\title{
Energy Loss versus Shadowing in the Drell-Yan Reaction on Nuclei
}

\author{
M.B. Johnson ${ }^{a}$, B.Z. Kopeliovich ${ }^{b, c, d}$, I.K. Potashnikova ${ }^{b, d}$ \\ and \\ P.L. McGaughey ${ }^{a}$, J.M. Moss ${ }^{a}$, J.C. Peng ${ }^{a}$, G. Garvey ${ }^{a}$, M. Leitch ${ }^{a}$ \\ C.N. Brown ${ }^{e}$, D.M. Kaplan ${ }^{f, g}$ \\ ${ }^{a}$ Los Alamos National Laboratory, Los Alamos, NM 87545, USA \\ ${ }^{b}$ Max-Plank-Institut für Kernphysik, 69029 Heidelberg, Germany \\ ${ }^{c}$ Inst. für Theor. Phys., Universität Regensburg, 93040 Regensburg, Germany \\ ${ }^{d}$ Joint Institute for Nuclear Research, Dubna, 141980 Moscow Region, Russia \\ ${ }^{e}$ Fermi National Accelerator Laboratory, Batavia, IL 60510, USA \\ ${ }^{f}$ Illinois Institute of Technology, Chicago, IL 60616, USA \\ ${ }^{g}$ Northern Illinois University, DeKalb, IL 60115, USA
}

\begin{abstract}
We present a new analysis of the E772 and E866 experiments on the nuclear dependence of Drell-Yan (DY) lepton pair production resulting from the bombardment of ${ }^{2} \mathrm{H}, \mathrm{Be}, \mathrm{C}, \mathrm{Ca}, \mathrm{Fe}$, and $\mathrm{W}$ targets by $800 \mathrm{GeV} / \mathrm{c}$ protons at Fermilab. We employ a light-cone formulation of the DY reaction in the rest frame of the nucleus, where the dimuons detected at small values of Bjorken $x_{2} \ll 1$ may be considered to originate from the decay of a heavy photon radiated from an incident quark in a bremsstrahlung process. We infer the energy loss of the quark by examining the suppression of the nuclear-dependent DY ratios seen as a function of projectile momentum fraction $x_{1}$ and dimuon mass $M$. Shadowing, which also leads to nuclear suppression of dimuons, is calculated within the same approach employing the results of phenomenological fits to deep inelastic scattering data from HERA. The analysis yields $-d E / d z=2.73 \pm 0.37 \pm 0.5 \mathrm{GeV} / \mathrm{fm}$ for the rate of quark energy loss per unit path length, a value consistent with theoretical expectations including the effects of the inelastic interaction of the incident proton at the surface of the nucleus. This is the first observation of a nonzero energy loss effect in such experiments.
\end{abstract}




\section{Introduction}

Nuclei can serve as a unique tool to study the space-time development of the strong interaction during its early stages, which is inaccessible in collisions between individual hadrons. The Drell-Yan (DY) reaction [1] on nuclear targets provides, in particular, the possibility of probing the propagation of partons through nuclear matter in its ground state, with the produced lepton pair carrying away the desired information on the energy and transverse momentum of the parent projectile quark after it has traveled in the nucleus. In this paper, we are specifically interested in the determination of the rate of energy loss per unit length $\kappa=-d E / d z$ of a fast quark propagating through a nucleus. We believe that the DY reaction data provides the cleanest way to single out such energy-loss effects.

One might also consider using other reactions for identifying energy-loss effects, with deep-inelastic scattering (DIS) coming to mind as one possibility. However, experimentally [2, 3, 4, 5], it has proven difficult to identify the partonic energy loss, not to mention to specify how to best measure this potentially important phenomenon. The origin of the difficulty can be appreciated by considering the following experiment. Suppose one were to measure the nuclear (A) dependence of semi-inclusive DIS, with known 4-momentum transfer. In this experiment, the A dependence of the energy (momentum) carried by a hadron created by the struck quark is supposed to convey the information regarding parton energy loss. However, besides the vacuum (initiated by the DIS) and induced (due to multiple interactions of the struck parton) energy loss, one must also account for the hadronization parton $\rightarrow$ hadron. As a rule, this hadron is subject to absorption when it is produced inside the nucleus. The complicated dynamics of this (see [6] and the comparisons of the predictions to data [5]) makes it extremely difficult to single out the net effect of energy loss.

High- $p_{T}$ production of hadrons off nuclei is even more complicated, since it includes a convolution of the parton distribution in the incident hadron with the high- $p_{T}$ parton-parton scattering cross section. Multiple parton interactions inside the nuclear medium (Cronin effect) also makes the interpretation extremely complicated. Yet another reaction sensitive to energy loss is charmonium production off nuclei. The violation of $x_{2}$ scaling observed in the E772 experiment [7] has already suggested the presence of energy-loss effects. However, final state absorption and coherence effects (see [8]) do not allow a clear identification of energy loss in the data.

In the DY reaction, the ratios $R_{A / D}$ of the cross section for a heavy nucleus $A$ compared to a light one (the deuteron $D$, say), are particularly relevant for the study of parton energy loss in nuclei. This is especially true in the region of large values of the the longitudinal momentum fraction of the projectile hadron carried by the DY pair, where significant nuclear suppression is clearly seen in this ratio. In an early study [9], it was shown that a ratio such as $R_{A / D}$ would be sensitive to $\kappa$ because the fraction $x_{q}$ of the light-cone momentum of the incoming hadron $h$ carried by projectile quark $q$ shifts to smaller values in $h A$ collisions as a result of initial-state energy loss. In the case of the DY reaction, this shift $\Delta x_{q}$ suppresses the ratio $R_{A / D}$ because it results in the sampling of the parton distribution at larger $x_{q}$ (for the same momentum of the dilepton), where the projectile parton densities are smaller. Thus, the ratio $R_{A / D}$ must decrease towards large Feynman $x_{F} \rightarrow 1$ where energy loss leads 
to a strongest suppression.

At the same time, nuclear shadowing was observed in Ref. [9] to be a competing source of suppression at large $x_{F}$ (more specifically, small values of the momentum fraction $x_{2}$ of the target). As it is easy to mix up energy loss and shadowing, one must take special precautions to disentangle these two effects when analyzing experimental data.

The first Drell-Yan data suitable for such an analysis was obtained in Fermilab E772/E866 experiments. An analysis of the E772 data [10] was made in [11] ignoring shadowing and assuming that $\kappa$ rises linearly with energy. The latter assumption was criticized [12] for violating the Landau-Pomeranchuk principle.

A better analysis was performed recently by the the E866 collaboration using the E866 data [13]. The analysis of Vasiliev, et al., which attempted to improve on that of [11] by including shadowing, considered three scenarios for energy loss, one of which was the same as in 11. All three of these gave $\kappa$ consistent with essentially zero energy loss, in contrast to the value $\kappa \approx 1.5 \mathrm{Gev} / \mathrm{fm}$ found in [11]. Thus, shadowing was found in [13] to be the main source of nuclear suppression of the DY cross section at large $x_{F}$.

Our present work differs from the previous analyses in that we attempt to unambiguously separate shadowing and energy loss by calculating the shadowing correction to the DY data using theory. The concept of coherence length, which plays a key role in respecting the Landau-Pomeranchuk principle, is essential to this formulation. It is our belief that the procedure employed by Vasiliev, et al. overestimated the shadowing contribution and hence substantially underestimated $\kappa$. The reason is that the shadowing correction was taken from a phenomenological analysis [14] that had already attributed the suppression of $R_{A / D}$ observed in E772 data at large $x_{F}$ entirely to shadowing. Preliminary results of our analysis are reported in Ref. [15].

We describe the interplay between quark energy loss and shadowing by working in the target rest frame using the light-cone approach of Refs. 116, 17, 18, 19, where these are given as separate contributions to the DY cross section. The target rest frame formulation is discussed in Sect. 2.1, where we relate the DY process to projectile fluctuations containing the DY pair. In terms of such fluctuations, the DY reaction may be viewed as occurring when interactions with the target break the coherence of the fluctuation and free a DY pair, i.e. bringing it on its mass shell. Such an interpretation is quite different from the conventional partonic treatment of DY process as annihilation $\bar{q} q \rightarrow \bar{l}$. However, the partonic interpretation is known to be Lorentz noninvariant and vary from frame to frame.

Such a rest frame formulation bears a close analogy to the more familiar light-cone description of deep-inelastic scattering (DIS) at small $x$ [20]. In that case, the incident virtual photon develops a quark-antiquark fluctuation which is brought to its mass shell by the interaction with the target. Again, this picture looks very different from the conventional parton model interpretation in the Breit frame, where it is described as absorption of the incident virtual photon by a quark (antiquark), which in this case belongs to the target.

The two processes, DY and DIS are known to be closely related in the parton model via QCD factorization. It is not surprising that the rest frame descriptions of these reactions also look similar. One of the advantages of this approach is the clear and simple treatment of nuclear shadowing, which is described in terms of the usual Glauber-Gribov theory. 
Shadowing in both processes, DY and DIS, is controlled by the same universal color-dipole cross section [21], which is the cross section for freeing the fluctuations. The strategy used in present paper is to treat the dipole cross section phenomenologically by fitting it to DIS data and then verifying the theory by comparing it to DIS scattering data on nuclei in the shadowing region (where there is no danger to mix up shadowing with energy loss). Then one can safely predict shadowing for the DY process. Such a strategy is based on the universality of the dipole cross section, which is a manifestation of QCD factorization.

The lifetime of the fluctuations, or coherence length, is another crucial quantity for understanding how energy loss and shadowing occur in the target rest frame. The coherence length is discussed and calculated in Sect. 2.2. This turns out to be mainly a function of Bjorken $x_{2}$, with some corrections that dramatically deviate from QCD factorization towards the smallest $x_{2}$.

We discuss the use of the DY reaction as a probe for quark energy loss in Sect. 3. Section 3.1 gives an overview of model expectations for energy loss. The string model (Sect. 3.1.1) predicts a constant rate of energy loss following the first inelastic collision of the incident hadron on the nuclear surface. The magnitude of this is about the same as that of the string tension, $\kappa_{s} \approx 1 \mathrm{GeV} / \mathrm{fm}$. Multiple interactions of the quark in the nuclear medium lead to an additional induced energy loss whose rate rises linearly with the path length of the quark. Numerically this is a small correction to the dominant constant term. Similar effects follow from perturbative QCD (Sect. 3.1.2). The first inelastic interaction of the incident proton in the nuclear surface initiates a long-lasting gluon bremsstrahlung, providing a constant rate of energy loss of about the same value as that given by the string model. Energy loss induced by quark rescattering also rises quadratically with the length of the path and is of similar value as in the string model. These two sources of energy loss are complementary and must be added, giving an expected rate of energy loss of about $2 \mathrm{GeV} / \mathrm{fm}$.

In the target rest frame, the suppression of $R_{A / D}$ by $\kappa$ is associated with short-lived fluctuations in which the DY pair is released immediately after the interaction of the projectile quark with a target nucleon. The produced dilepton pair carries undisturbed information about the energy of the quark traversing the nucleus only for such fluctuations. An important, although simple, consideration that arises here is the determination of the length of the path in nuclear matter over which the parton in the initial state loses energy. It is demonstrated in Sect. 3.2 that the mean length of this path does not follow an $A^{1 / 3}$ dependence but is, in fact, quite short compared to the nuclear radius. The distribution over path length is calculated for different nuclei. Nuclear suppression caused by energy loss is then given as a convolution of the quark distribution function of the incident hadron, modified by energy loss, with the DY cross section for the quark-nucleon interaction (Sect. 3.3). The former is borrowed from phenomenological models successfully describing soft hadronic collisions (Sect. 3.3.1), while the latter is fitted to data from the E772 experiment on a deuterium target (Sect. 3.3.2).

The other consideration in understanding the suppression of $R_{A / D}$ is, as we have remarked, the shadowing process. The critical quantities for describing shadowing in this approach are the coherence length and the effective fluctuation-freeing cross section (averaged over different fluctuations). The effective cross section is calculated in Sect. 7 in terms 
of a color-dipole cross section that describes data from HERA for the proton structure function at high $Q^{2}$. The shadowing correction is then calculated in an eikonal description of the multiple scattering of the incident quark in terms of the color-dipole cross section. In Sect. 1 we also confirm explicitly our description of shadowing by comparing the theory to deep inelastic scattering data, where energy loss is not an issue. We make our determination of $\kappa$ from $R_{A / D}^{\text {expt }}\left(x_{1}, M^{2}\right)$ based on the theoretical description of the effects of shadowing in Sect. 5. We fit the parameter $\kappa$ to the entire set experimental data without preselection, and the result is in agreement with the expected value of $\kappa$.

We also performed a variety of tests to check the stability of our results. These tests are described in Sect. 5.2. In one, we examined the sensitivity of the rate of energy loss to the relative contribution of shadowed and nonshadowed events. We determined that selecting only those events with small $x_{2}$, where shadowing is the dominant effect, does not change the results of the fit. We also artificially enlarged or eliminated shadowing, corrected shadowing calculations for multiple interactions, etc., and found stable results. These tests also provide a scale for the systematic uncertainties in our analysis.

Section 5.3 is devoted to the important issue of the disagreement between our determination of the rate of energy loss and that of a previous analysis of the E866 data for the DY reaction [13], which detected no energy-loss effect. In Sect. 5.3.1 we discuss why the choice of the target rest frame is the natural one for formulating the theory of energy loss. In Sect. 5.3b we find that the different conclusions reached in Ref. [13 can be explained by the substantially different space-time variation in the DY reaction at small $x_{2}$ and the larger density of antiquarks at large $x_{2}$ employed in this work. We justify our own results based on detailed theoretical arguments.

In Sect. 6 we summarize the results of the present analysis.

\section{Drell-Yan reaction in the nuclear rest frame}

\section{$2.1 \quad \bar{q} q \rightarrow \overline{l l}$ annihilation or $q \rightarrow q \bar{l} l$ bremsstrahlung?}

In the target rest frame, the Drell-Yan reaction at small $x_{2}$ corresponds to the electromagnetic radiation of a lepton pair by a projectile quark (valence or sea, depending on the value of $x_{1}$ ), rather than to $q \bar{q} \rightarrow l \bar{l}$ annihilation [16]. In this case, the $l \bar{l}$ pair is imagined to be liberated from a virtual fluctuation of the projectile when it interacts with a nucleon of the nucleus. Two examples of Feynman diagrams contributing to this are shown in Fig. 11. The cross section corresponding to these and other diagrams not shown has a factorized form in the impact parameter representation [16, 17, 18],

$$
\frac{d \sigma_{D Y}^{q N}}{d M^{2} d \alpha}=\int d^{2} r_{T}\left|\Psi_{q \overline{l l}}\left(r_{T}, \alpha\right)\right|^{2} \sigma_{\bar{q} q}\left(\alpha r_{T}, s_{q N}\right)
$$

where $s_{q N}$ is the square of the center-of-mass energy of the quark-nucleon collision, $M$ is the dilepton effective mass, and $\Psi_{q \bar{l}}\left(r_{T}, \alpha\right)$ is the light-cone wave function of the $|q \bar{l} l\rangle$ Fock

component of the projectile quark. This wave function depends on both the transverse 

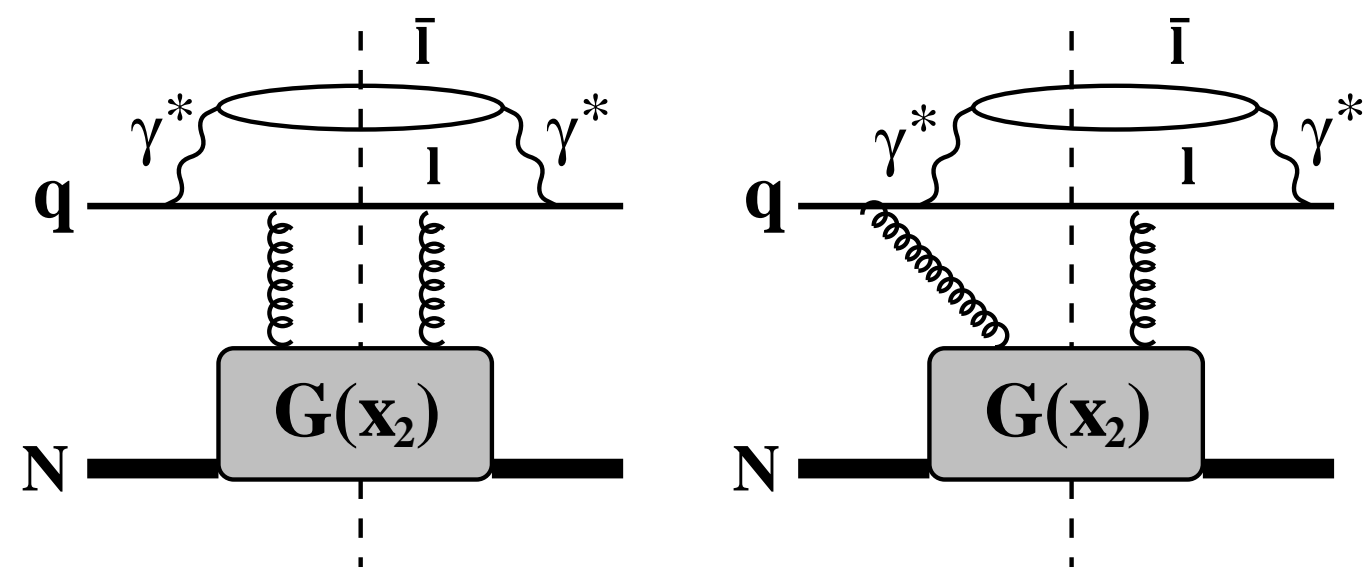

Figure 1: Feynman diagrams for the cross section of lepton pair production in a quark-nucleon collision with the $\gamma^{*}$ radiated before (left) and after (right) the interaction with the target.

separation $r_{T}$ between the $\bar{l}$ and the recoil quark and the fraction $\alpha$ of the initial light-cone momentum carried by the $\bar{l} l$ pair. Here, $\sigma_{\bar{q} q}(\rho, s)$ is the universal dipole cross section for the interaction of a colorless dipole $\bar{q} q$ with transverse separation $\rho=\alpha r_{T}$ from the nucleon, introduced in [21]. The appearance of the color dipole cross section in Eq. (11) arises from the difference between the interaction for $|q\rangle$ and $|q \bar{l} l\rangle$ fluctuations. This means, among other things, that the DY cross section receives no contribution if the transverse positions of the initial and final quarks coincide, and at small $\rho$ this cross section vanishes $\propto \rho^{2}$ [21]. Said otherwise, when $r_{T} \rightarrow 0$, the strong interaction cannot discriminate between the Fock components $|q\rangle$ and $|q \bar{l} l\rangle$ and therefore radiation is not possible.

As usual for bremsstrahlung, one cannot say whether the (virtual) photon is radiated before or after the interaction with the target (via gluon exchange); both are represented in Fig. 1. This quantum-mechanical uncertainty in the time of radiation is called the coherence time, which is the same as the coherence length since we are near the light cone. In terms of the light-cone approach, the coherence time can also be interpreted as the lifetime of the $|q \bar{l} l\rangle$ fluctuation of the incoming quark.

As already noted, one may distinguish this approach from the usual partonic interpretation of the DY reaction noting that the space-time development of high-energy reactions treated in terms of the parton model is not Lorentz invariant and depends on the reference frame. Indeed, since even the time ordering varies from frame to frame, the lepton pair produced at small values of Bjorken $x_{2} \ll 1$ in the DY reaction may be equivalently viewed as annihilation $\bar{q} q \rightarrow \bar{l} l$ in the rest frame of the photon [1] or as the decay of a heavy photon radiated in a bremsstrahlung process. 


\subsection{Coherence length}

The coherence length is an important quantity controlling nuclear effects in the DY reaction. If the coherence length is longer than the mean internucleon separation in the nucleus, different bound nucleons compete in freeing the $\bar{l} l$ fluctuation. This phenomenon is known as nuclear shadowing. On the other hand, if the coherence length is very short, the fluctuation has time to interact with only one bound nucleon. In this case, all nucleons contribute equally to the DY cross section, i.e. there is no shadowing.

The coherence length for a fluctuation of a projectile quark $q \rightarrow \overline{l l} q$ is given by the energy denominator,

$$
l_{c}^{D Y}=\frac{p_{q}^{+}}{M_{\bar{l} l q}^{2}-m_{q}^{2}}
$$

where

$$
M_{\overline{l l} q}^{2}=\frac{M^{2}}{1-\alpha}+\frac{m_{q}^{2}}{\alpha}+\frac{k_{T}^{2}}{\alpha(1-\alpha)}
$$

is the effective mass squared of the $\bar{l} l q$ fluctuation; $p_{q}^{+}=E_{q}+p_{q}^{\|}$is the light-cone momentum

of the incident quark; $M$ is the dilepton effective mass; and, $\vec{k}_{T}$ and $\alpha$ are the transverse momentum and fraction of the light-cone momentum of the parent quark carried by the $\bar{l} l$ pair, respectively.

If energy is conserved, the longitudinal momentum transfer between the initial state $|q\rangle$ and the fluctuation $|q \bar{l} l\rangle$ is $q_{c}=1 / l_{c}$. Thus, one can say that the coherence length is the maximal longitudinal distance between fluctuations that are in phase.

The DY variables $x_{1}$ and $x_{2}$ satisfy the equations,

$$
\begin{gathered}
x_{1} x_{2}=\frac{M^{2}}{s}, \\
x_{1}-x_{2}=x_{F},
\end{gathered}
$$

where $x_{F}$ is the Feynman variable, and they are interpreted in the parton model as the Bjorken variables of the annihilating quark and antiquark. They can equivalently be defined as fractions of the light-cone momenta $P^{ \pm}=E \pm P^{\|}$of the beam (b) and a target (t) nucleon carried by the lepton pair as follows,

$$
x_{1}=\frac{p_{\bar{l} l}^{+}}{P_{b}^{+}}, \quad x_{2}=\frac{p_{\overline{l l}}^{-}}{P_{t}^{-}} .
$$

Therefore $p_{\overline{l l}}^{+}=\alpha p_{q}^{+}=x_{1} P_{b}^{+}$, and the coherence length (2) averaged over $\alpha$ and $k_{T}$ reads

$$
\left\langle l_{c}^{D Y}\right\rangle=\frac{\left\langle K^{D Y}\right\rangle_{M^{2}, x_{1}}}{m_{N} x_{2}},
$$

where $\left\langle K^{D Y}\right\rangle_{M^{2}, x_{1}}$ is

$$
\left\langle K^{D Y}\right\rangle_{M^{2}, x_{1}} \equiv\left\langle K^{D Y}\left(\alpha, k_{T}\right)\right\rangle_{\alpha, k_{T}}=\left\langle\frac{M^{2}(1-\alpha)}{M^{2}(1-\alpha)+\alpha^{2} m_{q}^{2}+k_{T}^{2}}\right\rangle_{\alpha, k_{T}}
$$


In perturbative calculations, one would take $m_{q}$ to be the current quark mass. However, this is unsatisfactory in the present case because it leads to large transverse separations between the parent and recoil quark when the radiated dilepton takes essentially the entire initial quark momentum $M^{2}(1-\alpha) \sim m_{q}^{2}$. Indeed, in this case the separation becomes $r_{T} \sim 1 / m_{q}$, and one can grossly overestimate the contribution of large separations when using the approximation $\sigma_{\bar{q} q}\left(r_{T}\right) \propto r_{T}^{2}$. This divergence can be regularized by introducing an effective quark mass that suppresses the probability of large separations. Experience with DIS [23] shows that employing $m_{q} \approx 0.2 \mathrm{GeV}$ gives a good description of DIS data. We use the same effective mass in what follows for the DY reaction. Of course, this divergence does not create any problem when the dipole cross section saturates at large separations.

Clearly the coherence length, which controls nuclear shadowing, may not scale in $x_{2}$ and differs from the usually-used approximation $l_{c}=1 / 2 x_{2} m_{N}$. Moreover, it follows from (8) that as $x_{1} \rightarrow 1, l_{c} \rightarrow 0$ (since $\alpha>x_{1}$ ), i.e. nuclear shadowing vanishes. This fact is at variance with the usual expectation that shadowing increases towards the limit $x_{1}=1$, which corresponds to the smallest $x_{2}$. Although this contradiction shows that QCD factorization breaks down dramatically at large $x_{1}$, the result is quite consistent with the observation that all physics becomes soft [22] at large $x_{1}$.

The averaging in (\&) should be weighted by the light-cone wave function of the $q \bar{l} l$ fluctuation squared, which is known to diverge (for transversely polarized virtual photons) at small transverse $q-\bar{l} l$ separation $r_{T}$, i.e. for large $k_{T}$ 18 (similar to the case of deep inelastic scattering (DIS) for the fluctuation $\gamma^{*} \rightarrow \bar{q} q$ ). Such fluctuations with small $r_{T}$ do not interact; therefore, to avoid the nonsensical result that $\left\langle K^{D Y}\right\rangle \equiv 0$, which would arise from the fact that the vacuum fluctuations are dominated by large $k_{T}$ - leading to a divergent normalization - one also needs to include $\sigma_{\bar{q} q}\left(r_{T}, s\right)$ [16, 18] in the evaluation of Eq. (8). It is also necessary to include it here since we are interested only in those fluctuations that participate in the interaction. The dipole cross section plays the same role in Eq. (1), namely it prevents the interaction from discriminating between the fluctuation $|q\rangle$ and $|q \bar{l} l\rangle$ as $r_{T} \rightarrow 0$. A distinction between the two Fock components is clearly needed in order to liberate the $\bar{l} l$ pair.

In what follows, we are interested in nuclear shadowing, which in lowest order originates from double scattering. For reasons given above, the relevant mean coherence length should be weighted with $\sigma_{\bar{q} q}^{2}\left(\alpha r_{T}, s\right)$. Thus, Eq. (8) can be written explicitly as,

$$
\left\langle K^{D Y}\right\rangle_{M^{2}, x_{1}}=\frac{\int_{x_{1}}^{1} d x_{q} F_{q}^{h}\left(x_{q}\right) \int d^{2} k_{T}\left|\widetilde{\Psi}_{\overline{l l} q}\left(x_{1} / x_{q}, k_{T}\right)\right|^{2} K^{D Y}\left(x_{1} / x_{q}, k_{T}\right)}{\int_{x_{1}}^{1} d x_{q} F_{q}^{h}\left(x_{q}\right) \int d^{2} k_{T}\left|\widetilde{\Psi}_{\overline{l l} q}\left(x_{1} / x_{q}, k_{T}\right)\right|^{2}},
$$

where $F_{q}^{h}\left(x_{q}\right)$ is the quark distribution function of the beam hadron, which depends on the fraction $x_{q}$ of the hadron light-cone momentum carried by the quark. Hereafter we restrict ourselves to the transversely polarized $\bar{l} l$ pairs dominating the DY cross section, noting that the contribution of longitudinal polarization vanishes in any case as $x_{1} \rightarrow 1$. The modified 
light-cone distribution amplitude $\widetilde{\Psi}_{\bar{l} l q}^{T}$ reads 23

$$
\widetilde{\Psi}_{\overline{l l} q}^{T}\left(\alpha, k_{T}\right)=\int d^{2} r_{T} e^{i \vec{r}_{T} \cdot \vec{k}_{T}} \sigma_{\bar{q} q}\left(\alpha r_{T}, s\right) \Psi \frac{T}{l l q}\left(\alpha, r_{T}\right)
$$

where [16, 17, 18]

$$
\Psi_{l l q}^{T}\left(\alpha, k_{T}\right)=Z_{q} \frac{\sqrt{\alpha_{e m}}}{2 \pi} \chi_{f} \widehat{O}^{T} \chi_{i} K_{0}\left(\tau r_{T}\right) .
$$

Here $\chi_{i, f}$ are the spinors of the initial and final quarks, $Z_{q}$ is their charge, $K_{0}(x)$ is a modified Bessel function, and

$$
\tau^{2}=(1-\alpha) M^{2}+\alpha^{2} m_{q}^{2}
$$

The operator $\widehat{O}^{T}$ has the form

$$
\widehat{O}^{T}=i m_{q} \alpha^{2} \overrightarrow{e^{*}} \cdot(\vec{n} \times \vec{\sigma})+\alpha \overrightarrow{e^{*}} \cdot(\vec{\sigma} \times \vec{\nabla})-i(2-\alpha) \overrightarrow{e^{*}} \cdot \vec{\nabla},
$$

where $\vec{e}$ is the polarization vector of the $\bar{l}$ (virtual photon), $\vec{n}$ is the unit vector along the projectile momentum of the quark, and $\vec{\nabla}$ acts on $\vec{r}_{T}$.

To simplify calculations we use the dipole cross section in the form corresponding to small $r_{T}$ (a more realistic shape, leveling off at large $r_{T}$ [24, 25], leads to similar results),

$$
\sigma_{\bar{q} q}\left(r_{T}, s\right)=C(s) r_{T}^{2}
$$

where the constant of proportionality $C(s)$ does not enter into the final results. Then, the modified distribution amplitude in $k_{T}$-representation has the form,

$$
\widetilde{\Psi}_{\overline{l l} q}^{T}\left(\alpha, k_{T}\right)=2 Z_{q} \sqrt{\alpha_{e m}} C(s) \vec{e} \cdot \vec{k}_{T} \frac{i \alpha^{2} \tau^{2}}{\pi\left(\tau^{2}+k_{T}^{2}\right)^{3}},
$$

and the integration over $k_{T}$ in (9) can be performed analytically,

$$
\left\langle K^{D Y}\right\rangle=\frac{2 M^{2}}{3} \frac{N^{D Y}}{D^{D Y}}
$$

where

$$
\begin{gathered}
N^{D Y}=\int_{x_{1}}^{1} d \alpha F_{q}^{h}\left(x_{1} / \alpha\right) \frac{(1-\alpha) \alpha^{2}\left[1+(1-\alpha)^{2}\right]}{\tau^{6}} ; \\
D^{D Y}=\int_{x_{1}}^{1} d \alpha F_{q}^{h}\left(x_{1} / \alpha\right) \frac{\alpha^{2}\left[1+(1-\alpha)^{2}\right]}{\tau^{4}} .
\end{gathered}
$$

The coherence length $l_{c}^{D Y}$ calculated with (7) and (16) - (18) is plotted by solid curves as function of $x_{1}$ in Fig. 2 for different dilepton masses $M=4,5, \ldots 8 \mathrm{GeV}$.

Equation (16) should be compared with the analogous factor $\left\langle K^{D I S}\right\rangle=m_{N} x_{2}\left\langle l_{c}^{D I S}\right\rangle$ for DIS calculated in Ref. [23,

$$
\left\langle K^{D I S}\right\rangle=\frac{2 Q^{2}}{3} \frac{N^{D I S}}{D^{D I S}}
$$




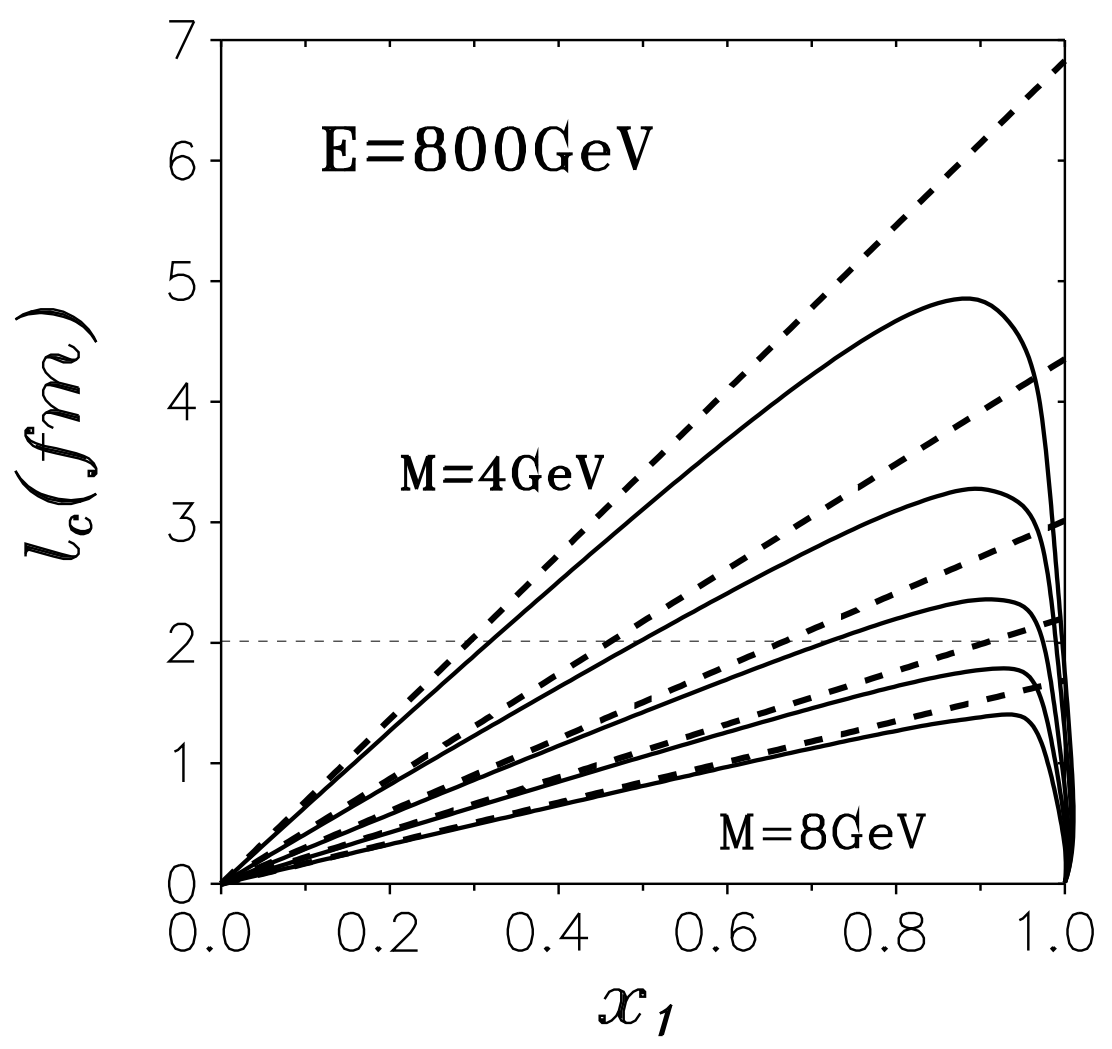

Figure 2: The mean coherence length (17) as function of $x_{1}$ and dimuon effective mass $M=4,5, \ldots 8 \mathrm{GeV}$ (solid curves). Dashed lines show the predictions of $Q C D$ factorization which relates the cross section of the $D Y$ reaction and DIS on a nucleus.

where

$$
\begin{gathered}
N^{D I S}=\int_{0}^{1} d \alpha \frac{\alpha(1-\alpha)\left[\alpha^{2}+(1-\alpha)^{2}\right]}{\epsilon^{6}} \\
D^{D I S}=\int_{0}^{1} d \alpha \frac{\alpha^{2}+(1-\alpha)^{2}}{\epsilon^{4}},
\end{gathered}
$$

and where

$$
\epsilon^{2}=\alpha(1-\alpha) Q^{2}+m_{q}^{2} .
$$

Obviously, $\left\langle K^{D I S}\right\rangle$ depends only on $Q^{2}$, while $\left\langle K^{D Y}\right\rangle$ depends on $M^{2}$ and $x_{1}$ and, as we have noted, vanishes as $x_{1} \rightarrow 1$. 
We compare in Fig. 目 the coherence length $\left\langle l_{c}\right\rangle=\langle K\rangle / m_{N} x_{2}$ calculated with (16) and (19) for kinematics corresponding to the E772/E866 experiments. The dashed curves are calculated for DIS at the same $x_{B j}=x_{2}$. The discrepancy between the two sets of curves, which increases towards $x_{1}=1$, manifests a deviation from factorization. Nevertheless, factorization is restored at small $x_{1}$ and/or at large $M$. Note that the factorization theorem requires only that the soft physics, which is common to DIS and DY, should factor from the reaction mechanism at large $Q^{2}$. Since shadowing in the distribution function is controlled in the target rest frame formulation by the coherence length and an effective cross section ( $\sigma_{\text {eff }}$ discussed in Sect. 1 below), factorization requires that these two quantities should also be the same for DIS and DY in this limit.

Note that the $x_{2}$ dependence of the mean coherence length is controlled mainly by the denominator in (17), while the factor $\left\langle K^{D Y}\right\rangle$ is a rather flat function of $x_{2}$ and $x_{1}$. This is demonstrated in Fig. 3 where $\left\langle l_{c}^{D Y}\right\rangle$ is plotted versus $x_{1}$ for different fixed values of $x_{2}$.

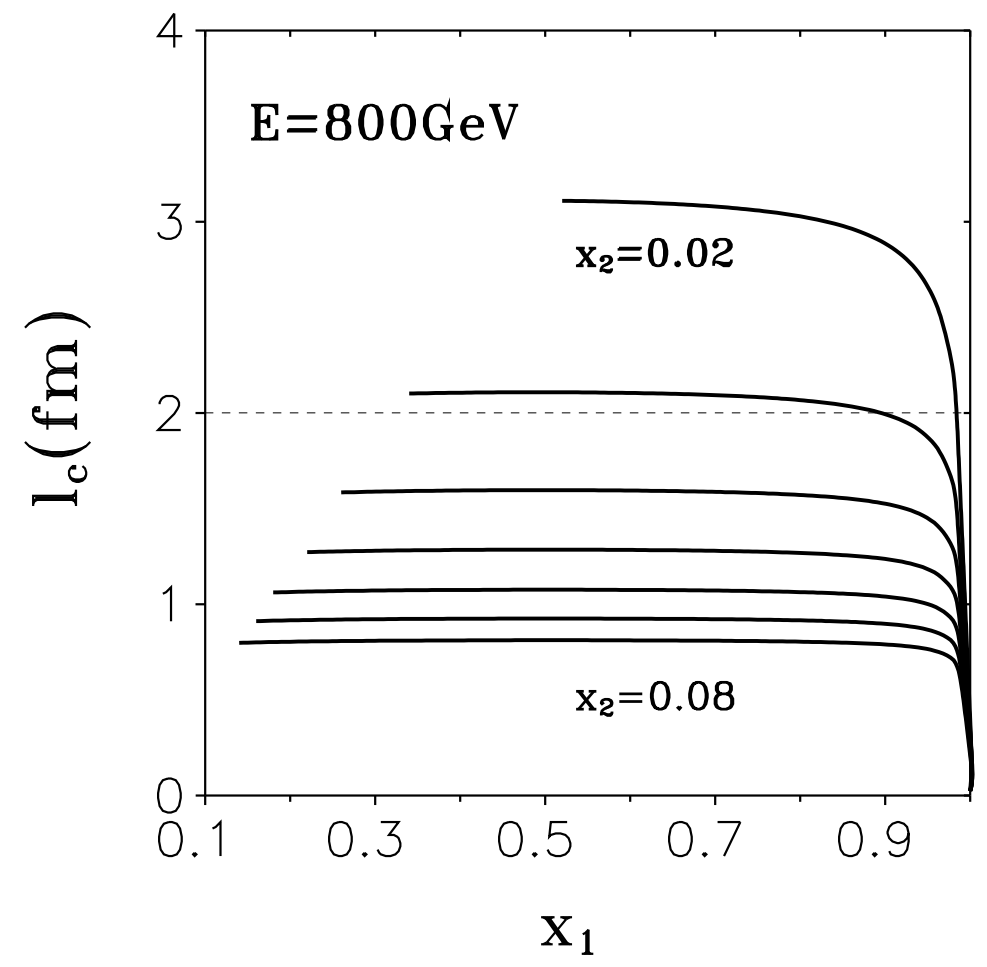

Figure 3: The mean coherence length (7) as function of $x_{1}$ at fixed values of $x_{2}=0.02,0.03, \ldots 0.08$.

At fixed $x_{2}$ the coherence length is nearly constant within the kinematical range of the E772/E866 experiments at $x_{1}<0.8$. The $x_{2}$ dependence of $\left\langle K^{D Y}\right\rangle_{x_{1}, x_{2}}$ at $x_{1}=0.7$, depicted in Fig. 1, demonstrates that $\left\langle K^{D Y}\right\rangle=0.2-0.3$ (see also in [23]) is quite small compared to the usually accepted $\left\langle K^{D Y}\right\rangle=0.5$. In the kinematical range of the E772/E866 experiments, $M>4 G e V$, and $\left\langle K^{D Y}\right\rangle \approx 0.3$ does not vary much. 


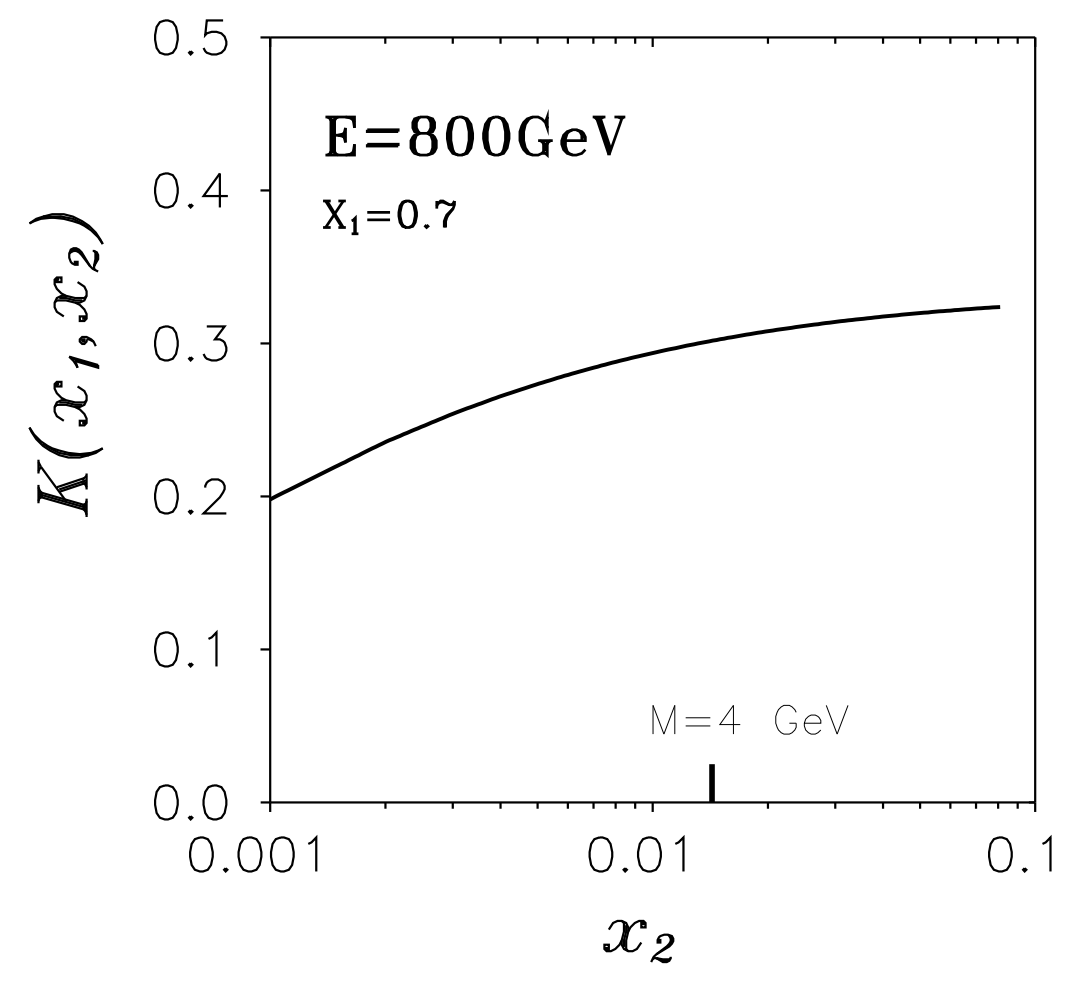

Figure 4: Factor $K^{D Y}$ appearing in the expression for $\left\langle l_{c}^{D Y}\right\rangle$ (Eq. (7)) as function of $x_{2}$ at $x_{1}=0.7$.

\section{$3 \quad$ Energy loss of the projectile quark in nuclear matter}

The coherence among the soft partons of the incident proton is destroyed as a result of inelastic interactions of the projectile in the surface of the nucleus. These partons then propagate through the nucleus independently, losing energy (to hadronization) and having their transverse momentum broadened by interactions. This is predominantly a nonperturbative process, since the projectile quark contains mainly soft fluctuations.

Eventually, one of these quarks may develop a hard fluctuation containing the lepton pair such that the lifetime of the fluctuation is short compared to the mean spacing between bound nucleons. The $\overline{l l}$ pair is immediately produced when a hard interaction occurs with a bound nucleon within the very short fluctuation time that we have referred to as the coherence time. Any change in the longitudinal or transverse momentum of the projectile quark caused by the preceding initial state interactions can diminish the energy of the projectile quark participating in the DY reaction. This affects the momentum spectrum for the produced DY pair, which then serves as a probe for the dynamics of the initial state interaction. It corresponds to the first of the two sources of nuclear suppression of $R_{A / D}\left(x_{F}\right)$ mentioned in the introduction. 


\subsection{Models for energy loss}

\subsubsection{String model}

The first inelastic interaction of the beam in the surface of the nucleus via color exchange with a bound nucleon leads to the formation of color strings between the target and beam partons. Due to the constant retarding action of the string, the leading projectile quark loses energy with a constant rate per unit length, $d E / d z=-\kappa_{s}$ [9, 26], which is invariant relative to longitudinal Lorentz boosts. The string tension $\kappa_{s}$ is related to the slope parameter $\alpha_{R}^{\prime}$ of the mesonic Regge trajectories [27] as

$$
\kappa_{s}=\frac{1}{2 \pi \alpha_{R}^{\prime}} \approx 1 \mathrm{GeV} / \mathrm{fm}
$$

This value imposes a scale for the expected rate of energy loss, which is independent of time. Correspondingly, the energy loss increases linearly with length $L$ of the path, $\Delta E=\kappa_{s} L$. The energy lost goes into acceleration of the target quarks and production of new hadrons.

In the simplest version of the string model, where at most one string can attach to a given quark, multiple interactions of the leading quark in the nuclear medium are expected to have no influence on its energy loss [28]. Indeed, no matter what happens to the leading quark before the hadronization is completed, it remains a color triplet and is slowed down with the same density of energy loss $\kappa_{s}$ by the color-triplet string attached to it: the quark is always being retarded with the same $\kappa_{s}$. Although such a conclusion sounds puzzling, it does not mean that multiple interactions in nuclear matter have no effect on hadronization. These interactions do make the fragmentation function of the quark softer, i.e. more particles are produced in the fragmentation region of the nucleus $\left(d n / d \eta \propto A^{1 / 3}\right)$ as the leading hadron diminishes its energy [9, 26]. This indeed may look like the result of energy loss, but it happens because each rescattering of the color string in nuclear matter initiates a new hadronization process from the very beginning, but with decreased initial energy [28].

The above treatment of the interaction is obviously oversimplified; in fact, multiple interactions in nuclear medium do induce extra energy loss. Indeed, the the valence quarks are surrounded by parton clouds, only a part of which can be resolved by a soft interaction. Multiple interactions in nuclear matter are obviously able to involve more of the partons. Correspondingly, the energy loss should grow more steeply than linearly in $L$.

This idea is realized in the dual parton model [29] (or quark-gluon string model [30]) by assuming that each multiple interaction in the nuclear medium activates a new sea $\bar{q} q$ pair, leading to the formation of an extra couple of color strings. The probabilities of multiple string production are given by the Abramovsky-Gribov-Kancheli (AGK) cutting rules [31]. The dual parton model skips over the space-time development of string production and decay, which takes long time proportional to the initial energy, jumping directly to the final spectrum of produced particles. We, however, are interested in the early stage of hadronization when the projectile quark is still propagating through the nucleus and its cloud of sea partons is still coherent. Therefore, we should follow the overall energy-loss of a nonperturbative constituent quark retarded by more than just one color string. 
A quark covering the distance $z$ in the nucleus, from the point of the first inelastic interaction of the incident hadron, loses an energy per unit length given by

$$
\frac{d E}{d z}=\kappa_{s}[1+\langle n(z)\rangle]
$$

where $\langle n(z)\rangle=\sigma^{q N} \rho_{A} z$ is the mean number of collisions experienced by the quark over the distance $z$. One can approximate the value of the nuclear density by $\rho_{A} \approx 0.16 \mathrm{fm}^{-3}$, since the path between the first inelastic collision and the DY pair production covers essentially only the interior of the nucleus. Correspondingly, the energy loss over a distance $L$ acquires a correction $\propto L$,

$$
\Delta E=\kappa_{s}\left(L+\frac{1}{2} \sigma^{q N} \rho_{A} L^{2}\right),
$$

The phenomenological quark-nucleon cross section is model dependent. In the additive quark model, $\sigma^{q N}=\sigma_{i n}^{N N} / 3 \approx 10 \mathrm{mb}$. The same value follows from the dual parton model [29, 30], which uses the weight factors $\sigma_{n}$ for the $n$-fold scattering appearing in the Glauber model as powers of $\sigma_{i n}^{N N}$. The new strings formed after each rescattering should be shared by three valence quarks.

In a more realistic model [32], the main part of the hadronic cross section corresponds to a soft nonperturbative interaction, which is unable to resolve and free the gluons that are located with small transverse separation $\left\langle r_{T}\right\rangle=r_{0}=0.3 \mathrm{fm}$ around the valence quarks [25]. This part of the cross section is independent of energy and does not obey quark additivity. Clearly, such soft interactions of the quark skeletons of the proton contribute to the linear term in (25). Only a semi-hard interaction is able to resolve the small-size gluon cloud and a correspondingly rather small quark-nucleon cross section related to the excitation of quark and gluon radiation. This process corresponds to the higher terms in topological expansion in the dual parton model, i.e. contributes to the $L^{2}$ term in (25). The predicted cross section is $\sigma^{q N}=(9 / 4) r_{0}^{2}\left(s / s_{0}\right)^{\Delta}$, where [32 $\Delta=0.17$ and $s_{0}=30 \mathrm{GeV}^{2}$. At $s=1600 \mathrm{GeV}^{2}$, one gets $\sigma^{q N}=4.2 \mathrm{mb}$, more than twice as small as the prediction of the additive quark model.

The mean values $\langle L\rangle$ and $\left\langle L^{2}\right\rangle$ in a heavy nucleus (with constant density) corrected for the mean free path $\lambda \approx 2 \mathrm{fm}$ of the incident proton in the nucleus are,

$$
\begin{aligned}
\langle L\rangle & =\frac{2}{3} R_{A}-\lambda \\
\left\langle L^{2}\right\rangle & =\frac{1}{2} R_{A}^{2}-\frac{4}{3} R_{A} \lambda+\lambda^{2} .
\end{aligned}
$$

For example, for $R_{A}=6 \mathrm{fm}$ the nonlinear $L^{2}$ correction in 25) is only $24 \%$ and $10 \%$ in the two models discussed above. It decreases dramatically, of course, for lighter nuclei. We neglect this small correction and assume for further applications that energy loss is a linear function of $L$.

\subsubsection{Perturbative QCD}

Although application of perturbative methods to soft processes is not completely legitimate, one may hope to get at least the scale of the effect by doing so. Energy loss treated 
perturbatively originates from gluon bremsstrahlung by a quark propagating through a medium. Here again one should distinguish between two sources of energy loss, namely gluon radiation originating in the first inelastic interaction, which occurs even in the vacuum, and gluon radiation induced by multiple interactions of the quark in the medium.

It was first demonstrated by Niedermayer 33] that as a consequence of coherence effects, (photon) gluon bremsstrahlung in the vacuum carries energy away with a constant rate,

$$
\Delta E(L)=E_{q} \int d^{2} k_{T} \int_{0}^{x_{\max }} d x x \frac{d n_{g}}{d x d k_{T}^{2}},
$$

where $\vec{k}_{T}$ and $x$ are the transverse momentum and fraction of the quark light-cone momentum of the parent quark momentum carried by the gluon, respectively. The upper integration limit $x_{\max }$ is fixed by the condition that all gluons contributing to $\Delta E$ are radiated within the path $L$. In order to be radiated, the gluon must lose coherence with the parent quark, otherwise one cannot disentangle between the quark plus its color field and the quark plus the radiated gluon. The distance $l_{f}$ over which this loss of coherence occurs and radiation formed is specified by the condition that the quark-gluon separation must exceed the transverse wave length of the gluon, which leads to $l_{f}=E_{q} x / k_{T}^{2}$, where $E_{q}$ is the quark energy [34]. Note that this value is twice as small as the coherence length defined in (2). Applying the condition $l_{f}<L$ we get $x_{\max }=L k_{T}^{2} / E_{q}$.

The spectrum of radiated gluons with $k_{T}^{2}$ smaller than the mean momentum transfer squared in the collision was calculated in [35],

$$
\frac{d n_{g}}{d x d^{2} k_{T}}=\frac{3 \alpha_{s}}{\pi^{2} x k_{T}^{2}} .
$$

Thus, the density of energy loss reads,

$$
-\frac{d E}{d z}=\frac{3 \alpha_{s}}{\pi}\left\langle k_{T}^{2}\right\rangle
$$

and a quark loses energy in the vacuum with a constant rate proportional to $\left\langle k_{T}^{2}\right\rangle$. Note that this is not the induced energy loss in a medium.

The proper value of $\alpha_{s} \approx 0.4$ for gluon radiation was calculated in [32. The transverse momentum squared of radiated gluons is rather large $k_{T} \approx 1 / r_{0}=650 \mathrm{MeV}$ [25, 32]. With these values we evaluate the energy loss rate (29) as $d E / d z \approx 0.8 \mathrm{GeV} / \mathrm{fm}$, which is amazingly close to the string tension. Note that the radiative energy loss is not supposed to be an alternative to what is given by the string model, but it is rather a different source that should be added to it. This is analogous to the additive contributions to the total hadronic cross section arising from nonperturbative strings and gluon radiation [32]

Quite a different situation arises if the quark originates from a hard reaction as, say, in DIS or a high- $p_{T}$ scattering event, where the rate of energy loss from gluon radiation is expected to be tremendous [6].

Another source of energy loss is induced gluon radiation due to multiple quark interactions in the medium. This has received much attention recently [34, 36], since it may serve 
as a probe for the production of dense matter in relativistic heavy ion collisions [36. The induced gluon radiation was calculated perturbatively [36], and it was concluded that the energy loss grows like $\Delta E \propto L^{2}$. This is a direct consequence of the relation (29) between the rate of energy loss and the transverse momentum squared of gluons, which follows that of the quarks and is known to rise $\propto L$. The relation between induced energy loss and nuclear broadening of the mean transverse momentum squared of the quark was established in [36],

$$
\Delta E=\frac{3 \alpha_{s}}{8} \Delta\left\langle p_{T}^{2}\right\rangle\langle L\rangle
$$

where the broadening of $\left\langle p_{T}^{2}\right\rangle$ was measured [37] to be rather small $\Delta\left\langle p_{T}^{2}\right\rangle \approx 0.1 G e V^{2}$ even for tungsten. Therefore the coefficient of $\langle L\rangle$ in (30) is about $0.075 \mathrm{GeV} / \mathrm{fm}$, an order of magnitude smaller than the string tension $\kappa_{s}$.

The induced energy loss of a nonperturbative quark, both the string and radiative parts, turn out to be a rather small fraction, less than $\sim 10-15 \%$, of the total energy loss even for heavy nuclei. Although this correction has a different $L$-dependence, one can effectively absorb it into the main linear term.

The dominant constant parts of the energy loss rate $d E / d z$ (so-called vacuum energy loss), related to the string tension (Sect. 3.1.2) and to gluon radiation, have different origins and, as we have said, should be added. The resulting rate of energy loss is thus expected to be

$$
-\frac{d E}{d z} \approx 2 G e V / f m
$$

\subsection{The quark path in the nucleus}

The DY reaction on a nuclear target is usually mediated by the debris resulting from an inelastic collision between the incident hadron and a bound nucleon on the front surface of a nucleus. This debris, once produced, propagates through the nucleus and produces the observed lepton pair when it strikes a bound nucleon, as illustrated in Fig. 5 (top). One would not expect to observe any difference between this and the DY reaction on a free nucleon for $z_{2}-z_{1} \rightarrow 0$. The reason is that the primordial momentum distribution of the projectile partons cannot be affected by soft inelastic interactions occuring over short times. However, when the distance $z_{2}-z_{1}$ is finite, the soft projectile partons may lose energy through hadronization and thus reach the point $z_{2}$ with diminished energy. However, at fixed $x_{1}$ the DY pair has to be produced with the same measured momentum, i.e. with an increased fraction of the initial momentum. As a result, the cross section for a DY pair produced with a given longitudinal momentum turns out to be smaller on a nucleus than it is on a free nucleon target.

It is usually incorrectly assumed that the quark propagates from the surface of the nucleus to the point where the DY pair is produced, which would mean that the mean quark path in the nucleus would be $\langle L\rangle \approx 3 R_{A} / 4$. According to Fig. 5, this should be shortened by at least the mean free path of a proton in a nucleus, $2 \mathrm{fm}$. This would substantially reduce $\langle L\rangle$, by a factor of two or more, so that the mean path between the point of DY pair production and the first inelastic interaction is actually shorter than the maximum possible 


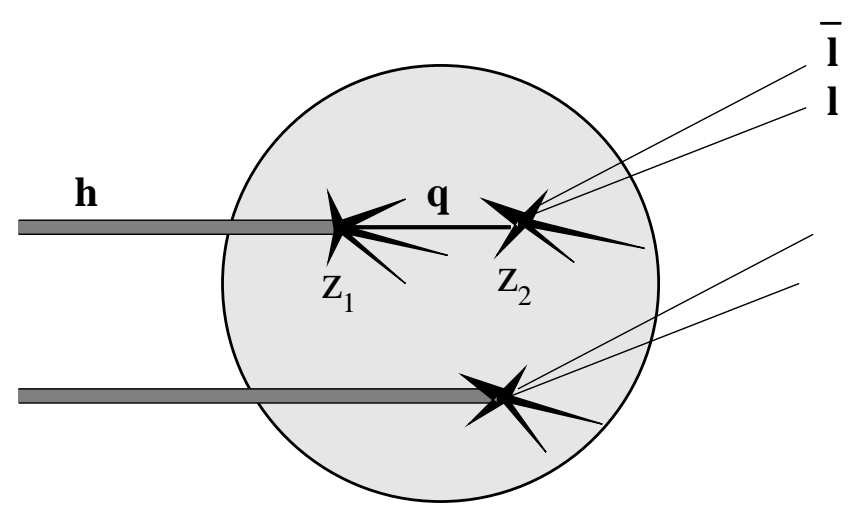

Figure 5: The space-time pattern for DY pair production off a nucleus. The upper example illustrates a case when the beam hadron experiences a soft inelastic interaction prior the hard interaction in which the $\bar{l} l$ pair is produced. The case where the DY pair is produced in the first inelastic interaction is illustrated at the bottom.

distance to the edge of the nucleus shown in Fig. 5. Additionally, there is some probability (dominant for light and medium-heavy nuclei) that the incident hadron has no interactions prior to DY pair production at point $z_{2}$. In order to find the mean path length $\langle L\rangle$ of the projectile quark in nuclear matter we should average $\left(z_{2}-z_{1}\right)$ in accordance with Fig. 5 , as done in [9],

$$
\begin{aligned}
\langle L\rangle & =\left(1-W_{0}\right) \frac{\sigma_{i n}^{h N}}{A} \int d^{2} b \int_{-\infty}^{\infty} d z_{2} \rho_{A}\left(b, z_{2}\right) \int_{-\infty}^{z_{2}} d z_{1} \rho_{A}\left(b, z_{1}\right)\left(z_{2}-z_{1}\right) \\
& \times \exp \left[-\sigma_{i n}^{h N} \int_{-\infty}^{z_{1}} d z \rho_{A}(b, z)\right] .
\end{aligned}
$$

The exponential factor requires that there is no inelastic interaction of the beam hadron prior point $z_{1}$. The probability of no inelastic interaction of the beam hadron in the nucleus prior the DY reaction (see the bottom part of Fig. 5) $W_{0}$ reads,

$$
W_{0}=\frac{1}{A \sigma_{i n}^{h N}} \int d^{2} b\left[1-e^{-\sigma_{i n}^{h N} T(b)}\right]=\frac{\sigma_{i n}^{h A}}{A \sigma_{i n}^{h N}} .
$$

We calculated $\langle L\rangle$ for a $p A$ collision taking $\sigma_{i n}^{p N}=30 m b$ and using a Woods-Saxon parameterization for the nuclear density [38]

$$
\rho_{A}(r)=\rho_{A}^{0}\left[1+\exp \left(\frac{r-R_{A}}{c}\right)\right]^{-1}
$$

where

$$
\rho_{A}^{0}=\frac{3 A}{4 \pi R_{A}^{3}}\left(1+\frac{\pi^{2} c^{2}}{R_{A}^{2}}\right)^{-1}
$$


and the nuclear radius $R_{A}$ and the edge thickness $c$ are fixed for each nucleus by a fit to data on electron-nucleus scattering [38].

As expected, $\langle L\rangle$ is much shorter than $3 R_{A} / 4$. Both of these results are plotted versus $A^{1 / 3}$ in Fig. 6. The corresponding probability distribution in $L$ is given by the expression

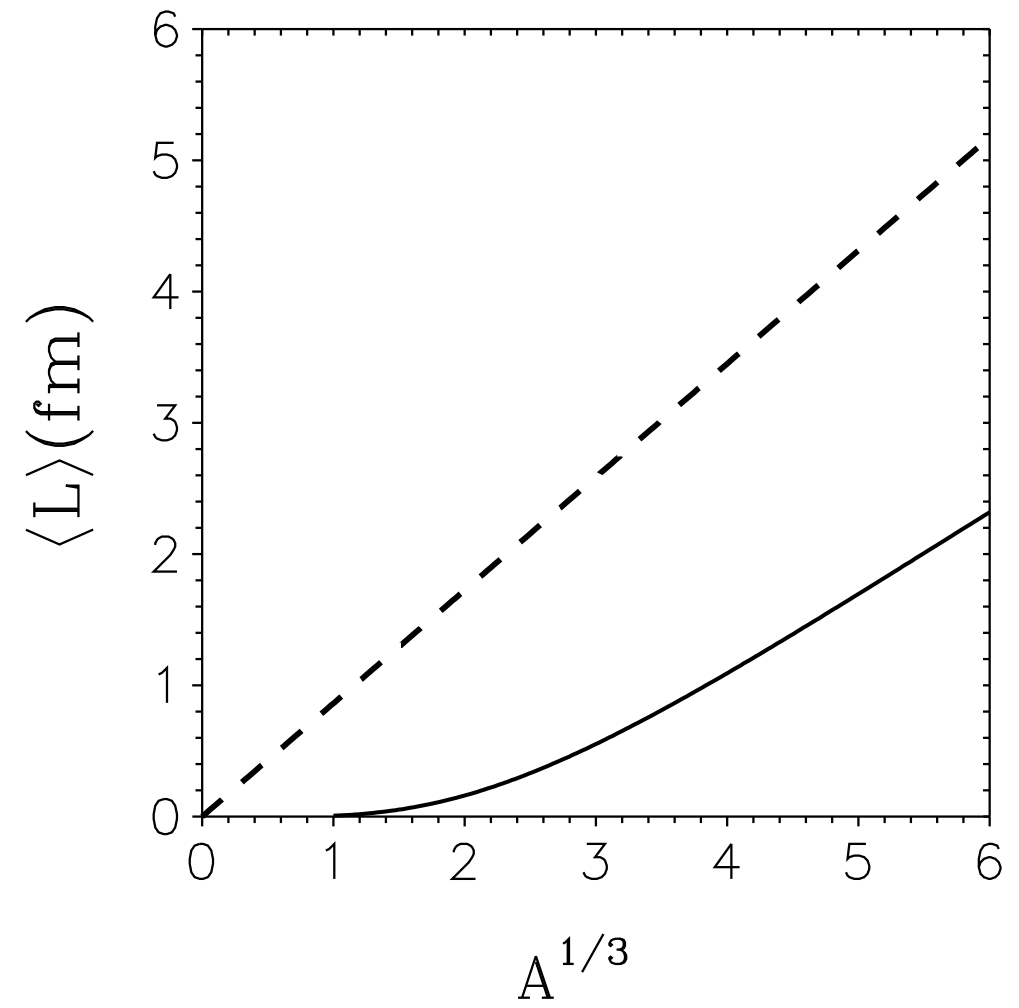

Figure 6: The mean length of the path of a quark between the points of the first inelastic interaction of the beam proton and DY pair production calculated with Eq. (32) as a function of $A^{1 / 3}$ (solid curve). The dashed curve shows the usual expectation $\langle L\rangle=3 R_{A} / 4$.

$$
W(L)=W_{0} \delta(L)+W_{1}(L)
$$

where

$$
\begin{aligned}
W_{1}(L) & =\frac{\sigma_{i n}^{h N}}{A} \int d^{2} b \int_{-\infty}^{\infty} d z_{2} \rho_{A}\left(b, z_{2}\right) \int_{-\infty}^{z_{2}} d z_{1} \rho_{A}\left(b, z_{1}\right) \delta\left(z_{2}-z_{1}-L\right) \\
& \times \exp \left[-\sigma_{i n}^{h N} \int_{-\infty}^{z_{1}} d z \rho_{A}(b, z)\right]
\end{aligned}
$$

which is normalized as $\int_{0}^{\infty} d L W_{1}(L)=1-W_{0}$. 
Although they are not as large, the effects of energy loss affect the DY cross section on a deuteron target as well. In this case the constant $W_{0}^{D}$ is given by the standard Glauber formula [39],

$$
W_{0}^{D}=1-\frac{\sigma_{t o t}^{N N}}{16 \pi} \int_{0}^{\infty} d q^{2} F_{D}\left(4 q^{2}\right) e^{-B_{e l}^{N N} q^{2}},
$$

where $B_{e l}^{N N} \approx 12 \mathrm{GeV}^{-2}$, and $F_{D}\left(q^{2}\right)$ is the deuteron charge formfactor parameterized and fitted in 40 to electron scattering data as

$$
F_{D}\left(q^{2}\right)=c_{1} e^{-b_{1} q^{2}}+c_{2} e^{-b_{2} q^{2}} .
$$

Here $c_{1}=0.55, c_{2}=0.45, b_{1}=19.66 \mathrm{GeV}^{-2}$, and $b_{2}=4.67 \mathrm{GeV}^{-2}$. This leads to $W_{0}^{D}=$ 0.96. Correspondingly, the distribution function $W_{1}(L)$ for a deuteron is given by the wave function squared related to the formfactor (39),

$$
\begin{aligned}
W_{1}^{D}(L) & =\left(1-W_{0}^{D}\right) \frac{L^{2}}{2 \sqrt{\pi}}\left[\frac{c_{1}}{\left(4 b_{1}+B_{e l}^{N N}\right)^{3 / 2}} \exp \left(-\frac{L^{2}}{4\left(4 b_{1}+B_{e l}^{N N}\right)}\right)\right. \\
& \left.+\frac{c_{2}}{\left(4 b_{2}+B_{e l}^{N N}\right)^{3 / 2}} \exp \left(-\frac{L^{2}}{4\left(4 b_{2}+B_{e l}^{N N}\right)}\right)\right] .
\end{aligned}
$$

\subsection{Nuclear suppression caused by energy loss}

Since the DY cross section decreases steeply as $\left(1-x_{1}\right)^{n}$ for $x_{1} \rightarrow 1$, any loss of energy by the quark by the time it reaches the point $z_{2}$ will result in a suppression of the DY cross section at large $x_{1}$. This effect was first suggested and estimated in [9]. In the more detailed approach taken here, we want to focus on the propagation and energy loss of the projectile quark that takes part in the DY reaction. In this case, the ratio $R_{A / N}$ of the DY cross sections of a nucleus to a nucleon can be represented as

$$
R_{A / N}^{\text {Eloss }}\left(x_{1}\right)=W_{0}+\frac{\int_{0}^{\infty} d L W_{1}(L) \int_{\left(x_{q}\right)_{\min }}^{1} d x_{q} F_{q}^{h}\left(x_{q}\right) d \sigma_{D Y}^{q N}\left(\widetilde{x}_{1}^{q}\right) / d \widetilde{x}_{1}^{q}}{\int_{x_{1}}^{1} d x_{q} F_{q}^{h}\left(x_{q}\right) d \sigma_{D Y}^{q N}\left(x_{1}^{q}\right) / d x_{1}^{q}} .
$$

Here $F_{q}^{h}\left(x_{q}\right)$ is the quark distribution function in the incident hadron; $x_{q}$ and $x_{1}^{q}=x_{1} / x_{q}$ are the fraction of the light-cone momentum of the incoming hadron $h$ carried by the quark and the fraction of the quark momentum carried by the $\bar{l} l$ pair, respectively. The lower integration limit is given by $\left(x_{q}\right)_{\min }=x_{1}+\Delta E / E_{h}$, and $\widetilde{x}_{1}^{q}=x_{1} /\left(x_{q}-\Delta E / E_{h}\right)$. According to the results of Sect. 3.1 we assume that the rate of energy loss $d E / d z=-\kappa$ is constant i.e. $\Delta E=\kappa L$. Note that as the cross section $d \sigma_{D Y}^{q N}\left(x_{1}^{q}\right) / d x_{1}^{q}$ in Eq. (41) corresponds to an incident constituent quark, while that in (1) corresponds to a current (perturbative) quark. To emphasize that these are different quantities, we use variable $x_{1}^{q}$ in (41) instead of $\alpha$ as in (11).

The energy loss $\Delta E$ varies due to fluctuations in the string tension, the number and energy of radiated gluons, and due the to the dependence of the distance $z_{2}-z_{1}$ shown in 
Fig. 5 on the distribution $W(L)$. We neglect the variation of $\kappa$, assuming $\kappa=$ const, and integrate the numerator of (41) over $\Delta E$ weighted by the distribution $W(\Delta E)$, with $W(L)$ given in (36).

The first term on the r.h.s. of Eq. (41) corresponds to the first term in (36), which represents the probability of no interaction preceding the DY reaction (the bottom part of of Fig. 5). The second term corresponds to one or more projectile interactions as illustrated in the upper part of Fig. 5 .

\subsubsection{Distribution of the valence quarks in the proton}

As mentioned above, in the limit of short coherence length $l_{c}^{D Y} \ll R_{A}$, the radiated photon arises from a hard DY fluctuation (one lasting for a short time) in the projectile quark, whereas the energy-loss mechanism is dominated by the softer fluctuations. Therefore, to calculate (41) we need to know the distribution function $F_{q}^{h}\left(x_{q}\right)$ and, correspondingly, the DY cross section $d \sigma_{D Y}^{q N}\left(x_{1}^{q}\right) / d x_{1}^{q}$ for soft quarks.

One should rely on a phenomenology of soft hadronic interactions to find the soft quark distribution function $F_{q}^{h}\left(x_{q}\right)$. The proper approach is found in the dual parton model [29] (quark-gluon string model [30]), which describes inelastic hadronic interactions via convolution of the quark distribution functions with the fragmentation functions of quarks to hadrons. The success of this phenomenology in describing the available experimental data on soft hadronic interactions justifies the quark distribution functions used above.

The proton quark distribution function in (41) includes contributions from the valence up and down quarks,

$$
F_{q}^{p}\left(x_{q}\right)=\frac{8}{9} F_{u}^{p}\left(x_{q}\right)+\frac{1}{9} F_{d}^{p}\left(x_{q}\right)
$$

where according to [29, 30],

$$
\begin{aligned}
& F_{u}^{p}\left(x_{q}\right)=N \frac{\left(1-x_{q}\right)^{1.5}}{\sqrt{x_{q}}}\left[1+\frac{6}{5} \frac{\sigma_{t o t}^{p N}}{8 \pi B^{p p}}\left(1-x_{q}\right)\right], \\
& F_{d}^{p}\left(x_{q}\right)=N \frac{6}{5} \frac{\left(1-x_{q}\right)^{2.5}}{\sqrt{x_{q}}}\left[1+\frac{8}{7} \frac{\sigma_{t o t}^{p N}}{8 \pi B^{p p}}\left(1-x_{q}\right)\right] .
\end{aligned}
$$

Here $N$ is a normalization factor inessential for (41); the factors $6 / 5$ and $8 / 7$ result from the normalization condition as well. The second terms in the square brackets in (43) and (44) are the first unitarity corrections (we neglect the small higher-order terms). They contain the total $p p$ cross section $\sigma_{\text {tot }}^{p p}=40 \mathrm{mb}$, the slope of elastic $p p$ scattering, $B^{p p}=12 \mathrm{GeV}^{-2}$, and an extra factor of 2 dictated by the AGK cutting rules [31. The behavior of these distribution functions as $x_{q} \rightarrow 0$ and as $x_{q} \rightarrow 1$ is dictated by Regge phenomenology and is related to the intercept of the leading meson trajectories $\alpha_{R}(0)=0.5$ and the nucleon trajectory $\alpha_{N}(0)=-0.5$ [29, 30]. 


\subsubsection{Quark-nucleon DY cross section}

In order to evaluate $R_{A / N}^{\text {eloss }}\left(x_{1}\right)$ in Eq. (41) we first of all have to fix the $x_{1}^{q}$ dependence of the quark-nucleon DY cross section. As was emphasized above, in the limit of short coherence length the quark that experiences initial state interactions is a constituent quark requiring the distribution function for soft quarks. Therefore, the DY quark-nucleon cross section $d \sigma_{D Y}^{q N} / d x_{1}^{q}$ should be treated correspondingly. Its shape cannot be predicted reliably since it depends in an essential way on the model for constituent quarks, which lies in the domain of nonperturbative effects. Instead, we parameterize the cross section in the form,

$$
\frac{d^{2} \sigma_{D Y}^{q N}}{d M^{2} d x_{1}^{q}}=K\left(M^{2}\right)\left(1-x_{1}^{q}\right)^{m}
$$

where $K\left(M^{2}\right)$ and $m$ are fitted parameters. We use data 41] for the DY cross section in $p+{ }^{2} H$ collisions and fit it by the expression

$$
\frac{d \sigma_{D Y}^{N N}}{d x_{1}} \propto \int_{x_{1}}^{1} d x_{q} F_{q}^{h}\left(x_{q}\right)\left(1-\frac{x_{1}}{x_{q}}\right)^{m}
$$

with $m=0.362 \pm 0.027$ taken to be independent of $M^{2}$. An example of the fit to the E772 data on deuterium target is depicted in Fig. 7 for the dimuon mass interval $7-8 \mathrm{GeV}$.

effects,

\section{Nuclear shadowing: DY versus DIS}

In the target rest frame, it is clear that the longitudinal momentum transfer to the nucleus in the DY reaction becomes small at high energies, in spite of the large effective mass $M$ of the dilepton. Therefore, different nucleons start interfering (destructively) in the production of the DY lepton pair, a phenomenon usually called shadowing.

Naively, one would not expect significant shadowing for hard reactions such as DIS, DY processes, heavy flavor production, etc., because these processes have tiny cross sections and because bound nucleons clearly do not shadow each other. However, substantial shadowing does exist for all of these, particularly the DY process, at high energies. The explanation is simple. First of all, shadowing arises from the soft components present in the hard reaction [16]. These soft components are small because the probability to develop a soft fluctuation in a hard reaction is small. However, this small size is compensated by a large interaction cross section. Once a soft fluctuation is created, the reaction is then driven by the subsequent hadronic interactions with the nucleus; these interactions are distinct from those producing the fluctuation to begin with and may be significant when the lifetime of the fluctuation (coherence time) is long.

The light-cone dipole representation of this reaction is especially suitable for the calculation of nuclear shadowing. The DY cross section in this representation has the form 


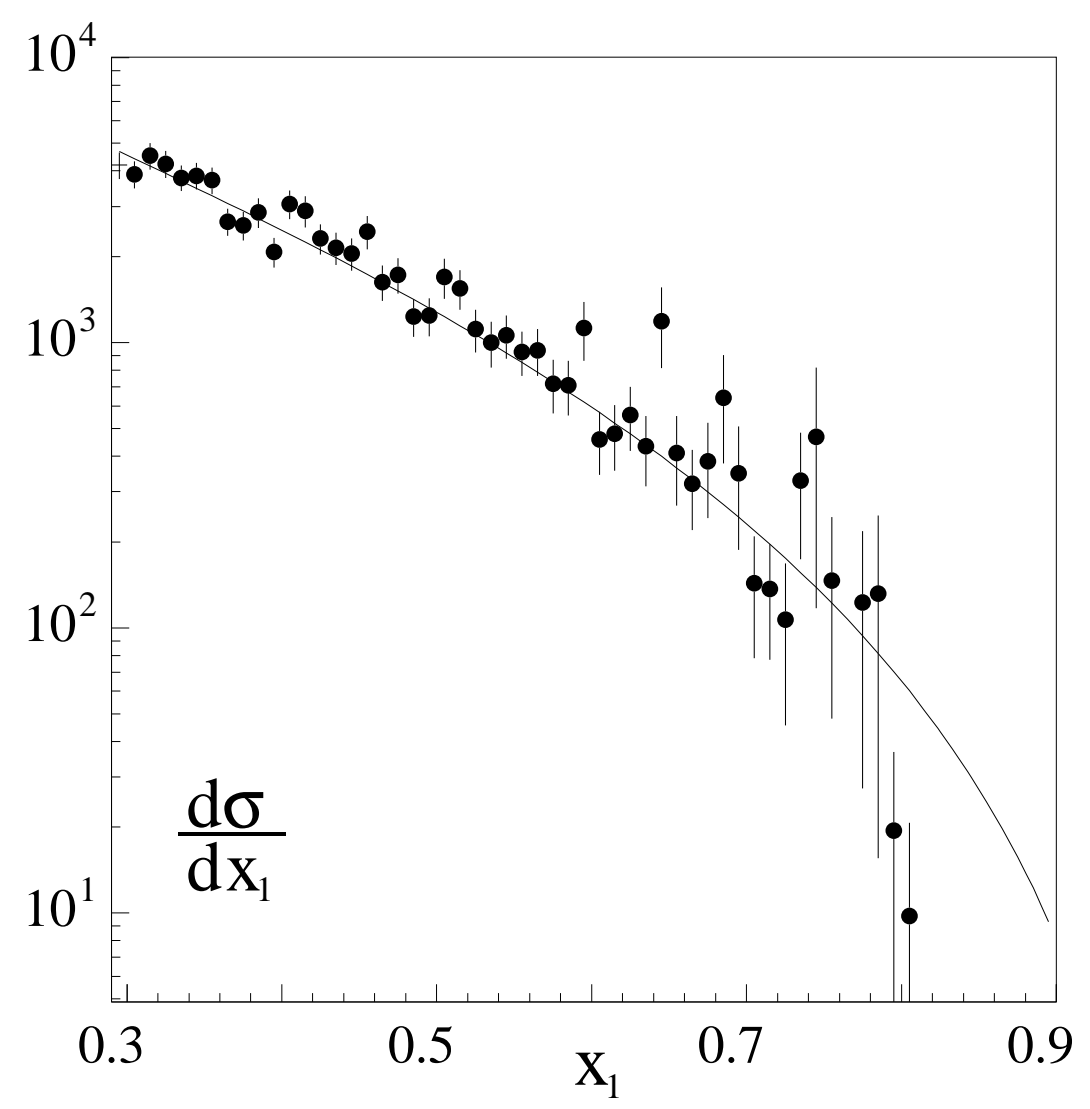

Figure 7: The cross section of the DY reaction on deuterium in arbitrary units as function of $x_{1}$. The data are from the E772 experiment for dimuon mass interval $7-8 \mathrm{GeV}$. The curve is the result of the fit $\propto$ $\left(1-x_{1}\right)^{0.362}$.

[16, 17, 18],

$$
M^{2} \frac{d \sigma_{N N}^{D Y}}{d M^{2} d x_{1}} \propto \int_{x_{1}}^{1} \frac{d \alpha}{\alpha^{2}} F_{q}^{p}\left(\frac{x_{1}}{\alpha}\right) \int d^{2} r_{T}\left|\Psi_{\bar{l} l q}\left(\alpha, \vec{r}_{T}\right)\right|^{2} \sigma_{\bar{q} q}\left(\alpha r_{T}\right),
$$

where $\Psi_{\overline{l l} q}\left(\alpha, \vec{r}_{T}\right)$ is defined in (11).

If $l_{c} \gg R_{A}$, the $q \bar{l} l$ projectile fluctuations are frozen by Lorentz time dilation during their propagation through the nucleus. Therefore, these fluctuations are eigenstates of the interaction and should have no inelastic corrections. One can simply eikonalize the dipole cross section in (47) in the case of a nuclear target [21, 16, 18],

$$
\sigma_{\bar{q} q}\left(\alpha r_{T}\right) \Rightarrow 2 \int d^{2} b\left\{1-\exp \left[-\frac{1}{2} \sigma_{\bar{q} q}\left(\alpha r_{T}\right) T(b)\right]\right\},
$$

where the averaging over $\alpha$ and $\vec{r}_{T}$ is performed in accordance with (47). In the case of weak shadowing, one can expand the exponent, dropping the higher-order shadowing corrections 
to obtain

$$
R_{A / N}^{s h a d}=\frac{\sigma_{A}^{D Y}}{A \sigma_{N}^{D Y}}=1-\frac{1}{4} \sigma_{e f f}\langle T\rangle+O\left(\left\langle\sigma^{3}\right\rangle\right)
$$

where

$$
\sigma_{e f f}\left(x_{1}, x_{2}, s\right)=\frac{\left\langle\sigma_{\bar{q} q}^{2}\left(\alpha r_{T}\right)\right\rangle}{\left\langle\sigma_{\bar{q} q}\left(\alpha r_{T}\right)\right\rangle}
$$

and where

$$
\langle T\rangle=\frac{1}{A} \int d^{2} b T^{2}(b)
$$

is the mean value of the nuclear thickness function

$$
T(b)=\int_{-\infty}^{\infty} d z \rho_{A}(b, z)
$$

with $\rho_{A}(r)$ the nuclear density distribution.

Note that naively one may expect a very small transverse size $\sim 1 / M^{2}$ for the $|q \bar{l} l\rangle$ fluctuations that define the dipole cross section in (48) and $\sigma_{\text {eff }}$. Although these fluctuations appear with a vanishing probability at large $M$, soft fluctuations with $\alpha \rightarrow 1$ can have a large transverse separation [16, 18], [see (11) - (12)]

$$
\left\langle r_{T}^{2}\right\rangle \sim \frac{1}{(1-\alpha) M^{2}}
$$

as follows from (11). For this reason, these soft fluctuations have a large interaction cross cross section and can make a sizable contribution to $\sigma_{e f f}$, in contrast to the harder ones 16 , 42 .

The transition region between the no-shadowing $\left(l_{c} \ll 2 f m\right.$, or $\left.x_{2} \sim 0.1\right)$ and asymptotic regimes $\left(l_{c} \gg\langle L\rangle\right.$, or $\left.1 / x_{2} \gg 3 m_{N} R_{A}\right)$, where Eq (48) is applicable, is most complicated and needs sophisticated calculations based on the path-integral approach [43, 23]. Nevertheless, if shadowing corrections are small (which is the case for the E772 and E866 data, where we face only the onset of shadowing), Eq. (49) can be easily interpolated [42,

$$
R_{A / N}^{s h a d} \approx 1-\frac{1}{4} \sigma_{e f f}\langle T\rangle F_{A}^{2}\left(q_{c}\right)
$$

where $F_{A}^{2}\left(q_{c}\right)$ is the longitudinal formfactor of the nucleus,

$$
F_{A}^{2}\left(q_{c}\right)=\frac{1}{\langle T\rangle} \int d^{2} b\left|\int_{-\infty}^{\infty} d z e^{i q_{c} z} \rho_{A}(b, z)\right|^{2} .
$$

Strictly speaking, this formfactor must also be involved in the averaging procedure for $\sigma_{\text {eff }}$ since $q_{c}=1 / l_{c}$ depends on $\alpha$ and $k_{T}$. In order to speed up the fitting procedure (see below) we replace $l_{c}$ by its mean value $\left\langle l_{c}\right\rangle$. Comparison with exact calculations for DIS done in Ref. [23] demonstrates that this approximation is sufficiently accurate. 
Beryllium is too light for the Woods-Saxon parametrization (34) of the nuclear density with which we describe heavier nuclei. Instead, we use for beryllium the harmonic oscillator density 38,

$$
\rho_{B e}(r)=\rho_{B e}^{0}\left(1+\alpha \frac{r^{2}}{a^{2}}\right) \exp \left(-\frac{r^{2}}{a^{2}}\right)
$$

where

$$
\rho_{B e}^{0}=\frac{9}{\left(\pi a^{2}\right)^{3 / 2}\left(1+\frac{3}{2} \alpha\right)}
$$

$a=1.77 \mathrm{fm}$, and $\alpha=0.631$.

The expression Eq. (55) is designed for medium and heavy nuclei and cannot be applied to the deuteron target involved in our analysis. In this case, one should use a different expression [39] [compare with (38)],

$$
R_{D / N}^{s h a d}=1-\frac{\sigma_{e f f}}{16 \pi} \int_{0}^{\infty} d q_{T}^{2} F_{D}\left(4 q^{2}\right)
$$

where $q^{2}=q_{T}^{2}+q_{c}^{2}$, with $q_{T}$ the transverse momentum. Using the deuteron charge formfactor $F_{D}\left(q^{2}\right)$ in the of form Eq. (39), we arrive at shadowing for a deuteron,

$$
R_{D / N}^{s h a d}=1-\frac{\sigma_{e f f}}{16 \pi}\left(\frac{c_{1}}{4 b_{1}} e^{-4 b_{1} q_{c}^{2}}+\frac{c_{2}}{4 b_{2}} e^{-4 b_{2} q_{c}^{2}}\right) .
$$

The effective cross section $\left\langle\sigma^{2}\right\rangle /\langle\sigma\rangle$ can be calculated by averaging in accordance with (47),

$$
\sigma_{e f f}\left(x_{1}, x_{2}, s\right)=\frac{\int_{x_{1}}^{1} d \alpha F_{q}^{p}\left(x_{1} / \alpha\right)\left[1+(1-\alpha)^{2}\right] \tau^{2} / \alpha^{2} \int d^{2} r_{T} K_{1}^{2}\left(\tau r_{T}\right) \sigma_{\bar{q} q}^{2}\left(\alpha r_{T}, x_{2}\right)}{\int_{x_{1}}^{1} d \alpha F_{q}^{p}\left(x_{1} / \alpha\right)\left[1+(1-\alpha)^{2}\right] \tau^{2} / \alpha^{2} \int d^{2} r_{T} K_{1}^{2}\left(\tau r_{T}\right) \sigma_{\bar{q} q}\left(\alpha r_{T}, x_{2}\right)}
$$

where $K_{1}(y)$ is the modified Bessel function and $\tau$ is defined in Eq. (12). It is reasonable to use here the phenomenological $x$-dependent dipole cross section [24] which describes very well data from HERA for the proton structure function at high $Q^{2}$,

$$
\sigma_{\bar{q} q}(\rho, x)=\sigma_{0}\left[1-\exp \left(-\frac{\rho^{2}}{\rho_{0}^{2}(x)}\right)\right]
$$

where $\sigma_{0}=23.03 \mathrm{mb}$ and $\rho_{0}(x)=0.4\left(x / x_{0}\right)^{0.144} \mathrm{fm}, x_{0}=3.04 \cdot 10^{-4}$. In the case of the DY reaction, $x=x_{2}$.

The results of the calculations for $\sigma_{e f f}\left(x_{1}, x_{2}, s\right)$ at $s=1600 \mathrm{GeV}^{2}$ are depicted in Fig. 8 as a function of $x_{1}$ for different fixed values of $x_{2}$. Note that the effective absorption cross section $\sigma_{\text {eff }}$ substantially increases with $x_{1}$. This is a manifestation of factorization breaking, since suppression (i.e. shadowing) of the nuclear structure function cannot increase with rising $M^{2}$ at fixed $x_{2}$. In the light-cone approach this behavior is easily interpreted: the mean transverse separation squared that enters into ([60), $\alpha^{2}\left\langle r_{T}^{2}\right\rangle \sim 1 / \tau^{2} \approx 1 /\left[M^{2}(1-\alpha)\right]$, 


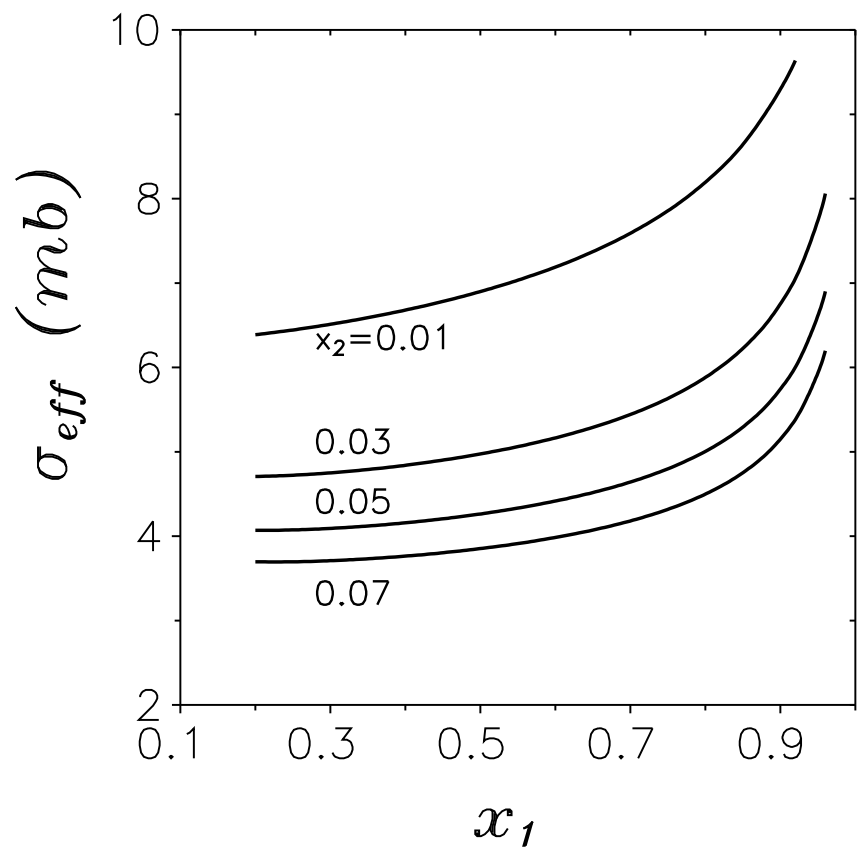

Figure 8: The effective cross section Eq. 60) as a function of $x_{1}$ for different fixed values of $x_{2}=0.02,0.03,0.05$ and 0.07 .

increases as $x_{1} \rightarrow 1$ because $\alpha>x_{1}$. Nevertheless, Fig. 8 shows that the $x_{1}$ dependence of $\sigma_{e f f}$ is quite weak for $x_{1}<0.8$.

Although we cannot test our calculations of shadowing by comparing them to nuclear suppression in DY reactions, which are affected by energy loss effects, we may check them against nuclear shadowing in DIS. In this case $\sigma_{\text {eff }}$ reads [23],

$$
\sigma_{e f f}^{D I S}\left(x_{2}, Q^{2}\right)=\frac{\int_{0}^{1} d \alpha\left[\alpha^{2}+(1-\alpha)^{2}\right] \epsilon^{2} \int d^{2} r_{T} K_{1}^{2}\left(\epsilon r_{T}\right) \sigma_{\bar{q} q}^{2}\left(r_{T}, x_{2}\right)}{\int_{0}^{1} d \alpha\left[\alpha^{2}+(1-\alpha)^{2}\right] \epsilon^{2} \int d^{2} r_{T} K_{1}^{2}\left(\epsilon r_{T}\right) \sigma_{\bar{q} q}\left(r_{T}, x_{2}\right)},
$$

where $\epsilon$ is introduced in (22).

The calculated $\sigma_{\text {eff }}^{D I S}\left(x_{2}, Q^{2}\right)$ turns out to be rather different from $\sigma_{\text {eff }}^{D Y}\left(x_{2}, Q^{2}\right)$ calculated with Eq. (60). Their ratio is plotted in Fig. 9 as a function of $x_{1}$ for different dilepton masses. The fact that the ratio is not unity is a deviation from factorization (except near $x_{1}=1$, where the factorization theorem does not apply since, as we have noted, all physics becomes soft here [22]). However, the ratio may be shown to approach unity logarithmically for large $M^{2}$ (and $x_{1}<1$ ), as required by the factorization theorem and as suggested by the results in the figure.

The effective absorption cross section controlling nuclear shadowing is related to forward diffractive dissociation [44]. As a simple test, we can compare our results for $\sigma_{\text {eff }}^{D I S}$ with data for diffractive dissociation of highly virtual photons which were measured at HERA to be 


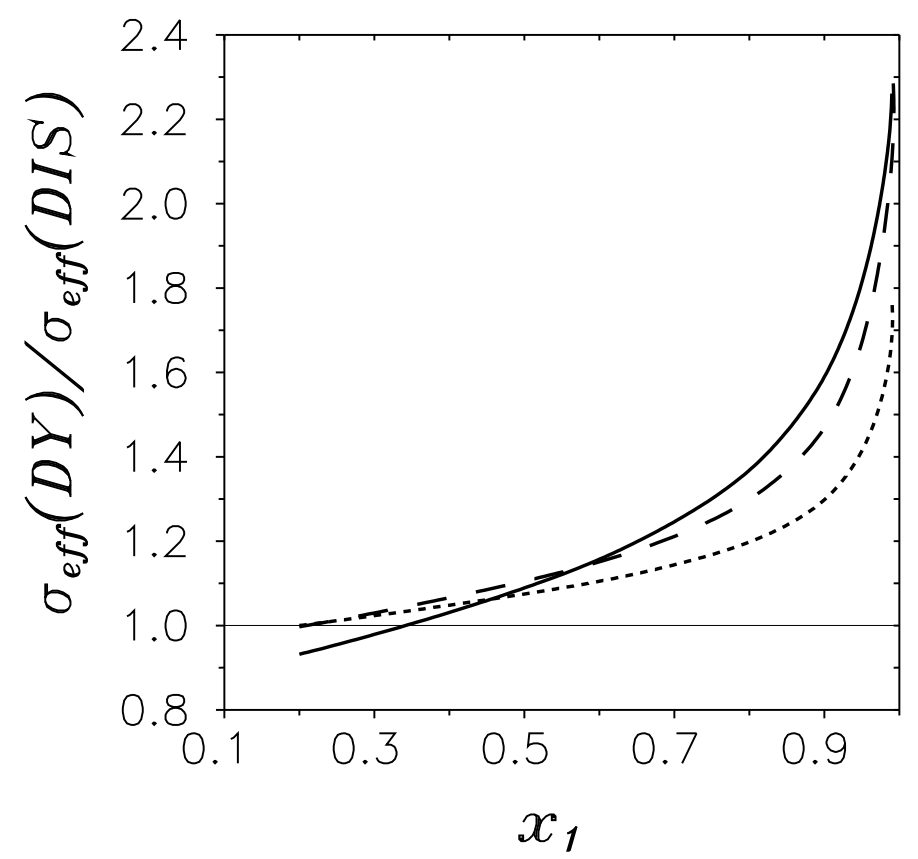

Figure 9: Ratio $\sigma_{\text {eff }}^{D Y} / \sigma_{\text {eff }}^{D I S}$ as a function of $x_{1}$ for $M=4$ (solidcurve), 20 (dashed) and $100 \mathrm{GeV}$ (dotted). Deviation from unity demonstrates breakdown of $Q C D$ factorization (see text).

about $10 \%$ of the total DIS cross section,

$$
\frac{\sigma_{d d}^{D I S}}{\sigma_{t o t}^{D I S}}=\frac{\sigma_{e f f}\left(x_{1}, x_{2}, s\right)}{16 \pi B_{d d}^{D I S}} \approx 0.1,
$$

where the subscript $d d$ means diffractive dissociation, and $B_{d d}^{D I S} \approx 5 \mathrm{GeV}^{-2}$.

A more rigorous test would involve a direct comparison of the calculated nuclear shadowing for DIS with data. Shadowing can be calculated using Eqs. (62) and (54), where the argument of the formfactor, $q_{c}=x m_{N}\left\langle K^{D I S}\right\rangle$, is given by Eq. (19) (see Fig. 2). The results are plotted in Fig. 10 as the dashed curve and compared with data from the NMC experiment. Since the value of $Q^{2}$ in the data varies with $x$ we incorporate this correlation in our calculations; we use the same set of $x$ and $Q^{2}$ as in the NMC data. Shadowing for the deuteron is also taken into account. At large $x \sim 0.1$, the data display about $2 \%$ antishadowing, the origin of which is poorly understood, although it is probably related to the same nuclear medium effects that cause the EMC effect. As this dynamics is not included in our calculations, it is not surprising that we underestimate the data in this region.

At smaller $x$ (and smaller $Q^{2}$ ), as expected, the calculated shadowing keeps decreasing and does not seem to saturate. This is due to the rise of $\sigma_{e f f}\left(x, Q^{2}\right)$ at small $x$ and $Q^{2}$.

At even smaller $x$, gluon shadowing also becomes important. The onset of gluon shadowing occurs only at $x<10^{-2}$ because it is related to fluctuations containing extra gluons that are heavier than $\bar{q} q$ fluctuations. Correspondingly, the coherence length for gluon shadowing 


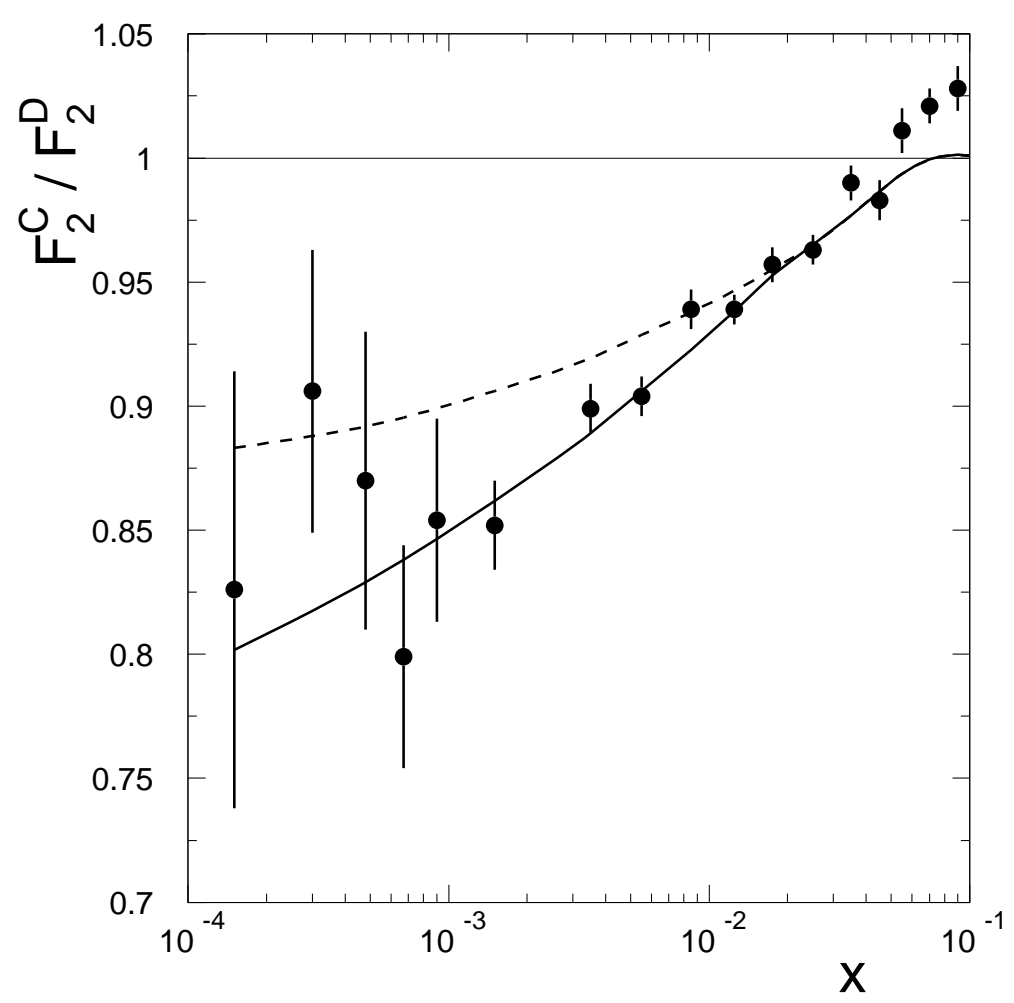

Figure 10: Normalized ratio of carbon to deuterium structure functions. The data are from the NMC experiment [45]. The dashed curve is calculated using Eqs. (54), (58), (62) for each data point at the same values of $x$ and $Q^{2}$. The solid curve includes also the effect of gluon shadowing as it is calculated in 25.

is shorter than it is for quarks [23]. Nuclear shadowing for gluons at small $Q^{2}$ relevant for the NMC data and at low $x$ is predicted in [25] (see Fig. 6 of that paper). We multiply $F_{2}^{C} / F_{2}^{D}$ by the gluon shadowing factor as calculated in [25]. The result shown by the solid curve is in good agreement with the data and confirms the reliability of our description of shadowing, which contains no free parameters.

\section{$5 \quad$ Energy loss versus shadowing}

By including shadowing explicitly in the analysis of the DY data, we are able to utilize the entire data set of the E772/E866 experiments for the ratio $R^{\exp }\left(x, M^{2}\right)$. This must be done recognizing that shadowing and energy loss are complementary sources of nuclear suppression. If the lifetime of a fluctuation containing the lepton pair substantially exceeds the size of the nucleus, we assume that the energy loss of the hadronic part of the fluctuation does not affect the spectrum of dileptons, which are created as a fluctuation long in advance 
of the nucleus. Thus, in the two limiting cases of very long or very short coherence time one finds either shadowing or energy loss present, correspondingly, not both together.

The DY amplitude in the long $l_{c}$ limit differs from that in the short $l_{c}$ limit by additional terms that account for interference between the DY amplitudes involving different numbers of nucleons. Within the approximation that the double scattering correction dominates, which is quite accurate if shadowing is small (appropriate for the kinematics of the E772/E866 experiments), the interference term always appears multiplied by the nuclear formfactor squared, as in Eq. (54). The transition from the short $l_{c}$ regime, described by Eq. (41), to the regime of $l_{c} \gg R_{A}$, described by Eq. (54), is likewise controlled by the nuclear formfactor. It is easy to write the interpolating expression, namely

$$
R_{A / N}\left(x_{1}, M^{2}\right)=\left[1-F_{A}^{2}\left(q_{c}\right)\right]\left[R_{A / N}^{\text {Eloss }}\left(x_{1}\right)-1\right]+R_{A / N}^{\text {shad }}\left(x_{1}, M^{2}\right)
$$

where $F_{A}^{2}\left(q_{c}\right)$ is given by (55) and $R_{A / N}^{\text {Eloss }}$ and $R_{A / N}^{\text {shad }}$ are given by (41) and (54) respectively. Obviously, (64) correctly reproduces the short and long coherence length limits and mixes these effects when both contribute. A nice feature of Eq. (64) is that the effect of energy loss is weakened with rising $l_{c}$ and eventually vanishes at $l_{c} \gg R_{A}$.

\section{$5.1 \quad$ Results}

With Eq. (64), we have adjusted $\kappa$ to fit the entire set of ratios $C / D, C a / D, F e / D, W / D$, $\mathrm{Fe} / \mathrm{Be}$ and $W / \mathrm{Be}$ from the E772 [10] and E866 [13] experiments double-binned in $x_{1} \geq 0.3$ and $M \geq 4 \mathrm{GeV}$. Since the normalization of the data is subject to systematic uncertainties, we included them in the fit by introducing for each experiment an overall normalization $N=1 \pm \Delta N$ for the theoretical values of the nuclear ratios. We allowed these to contribute to $\chi^{2}$ by treating them as additional experimental points and assuming a normal distribution for them. A few examples of the fit results are depicted in Figs. 11, 12, and 13 (more bins in $M$ and a greater variety of nuclei were involved in the fitting). In addition to the solid curves, which show the full result of the fit, we also show the contribution of shadowing alone in the dashed curves. Shadowing was calculated for the mean value of mass calculated for each interval as $\sqrt{\left\langle M^{2}\right\rangle}$.

We found the following value for the rate of energy loss,

$$
-\frac{d E}{d z}=2.73 \pm 0.37 \mathrm{GeV} / \mathrm{fm}
$$

with $\chi^{2} /$ d.o.f. $=0.9$, and normalization factors $N(E 772)=1.014 \pm 0.005$ and $N(E 866)=$ $0.996 \pm 0.006$.

\footnotetext{
${ }^{1}$ This is different for nuclear broadening of the transverse momentum of a DY pair. Since a nuclear target supplies a stronger kick to the quark part of the fluctuation compared to a nucleon target, the nucleus is able to break up the coherence of harder fluctuations, i.e. fluctuations with larger transverse momentum of the lepton pair [18].
} 

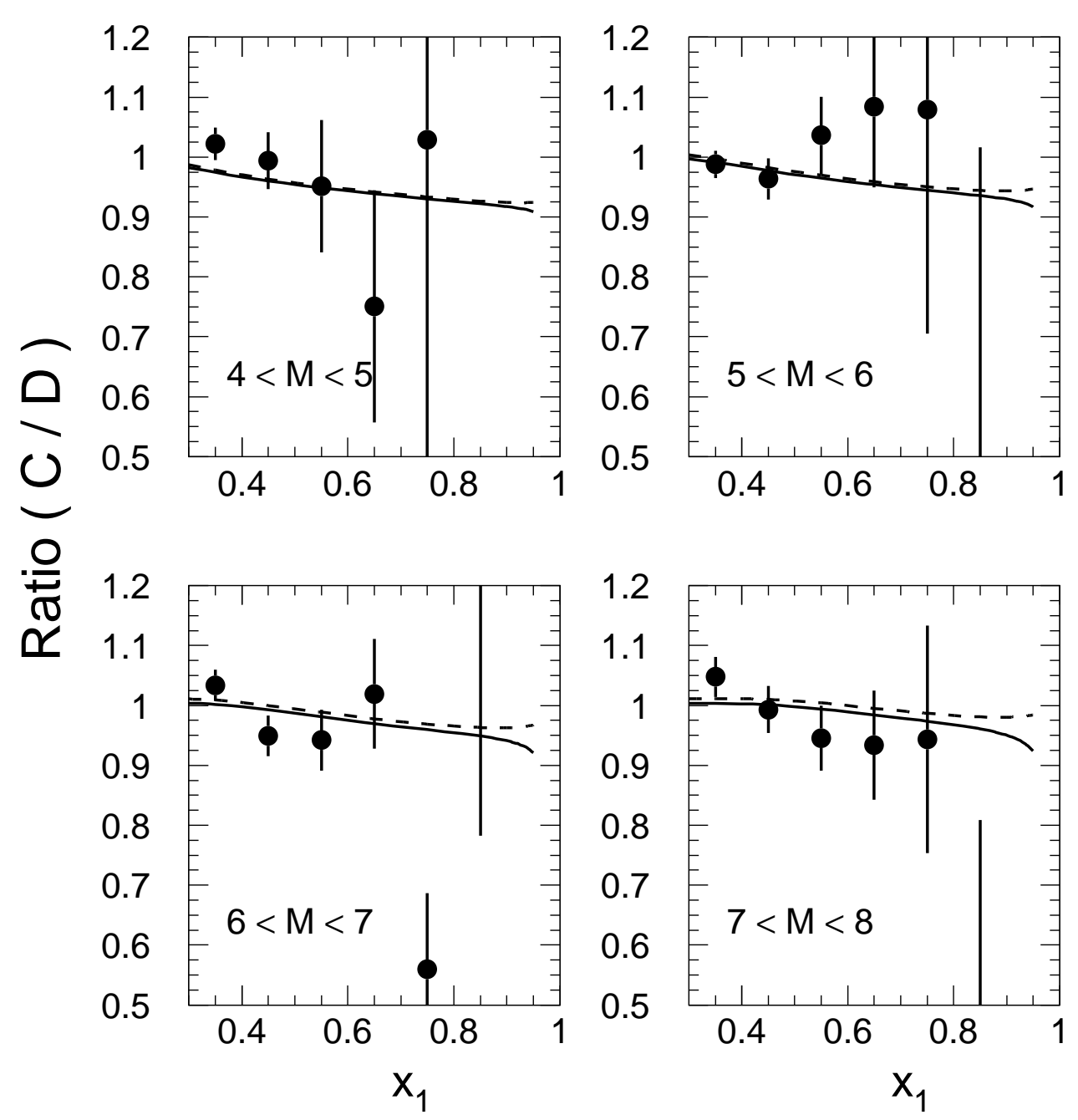

Figure 11: Examples of ratios of the DY cross sections on carbon to deuterium as functions of $x_{1}$ for various intervals of $M$. Dashed curves correspond to net shadowing contribution, solid curve show the full effect including shadowing and energy loss. Data from Refs. [10],46].

A nice feature of the fit is the consistency of the values of $d E / d z$ resulting from independent fits to the E722 [46] and E866 [47] data,

$$
\begin{aligned}
& -\left.\frac{d E}{d z}\right|_{E 772}=2.32 \pm 0.52 \mathrm{GeV} / \mathrm{fm} \\
& -\left.\frac{d E}{d z}\right|_{E 866}=3.14 \pm 0.53 \mathrm{GeV} / \mathrm{fm}
\end{aligned}
$$

with $\chi^{2} /$ d.o.f. $=0.85$ and $\chi^{2} /$ d.o.f. $=1.02$ for the E772 and E866 data respectively, and normalization factors $N(E 772)=1.01 \pm 0.006$ and $N(E 866)=1.00 \pm 0.007$.

The rate of energy loss given in Eq. (65) is larger than the value $1 \mathrm{GeV} / \mathrm{fm}$ suggested 

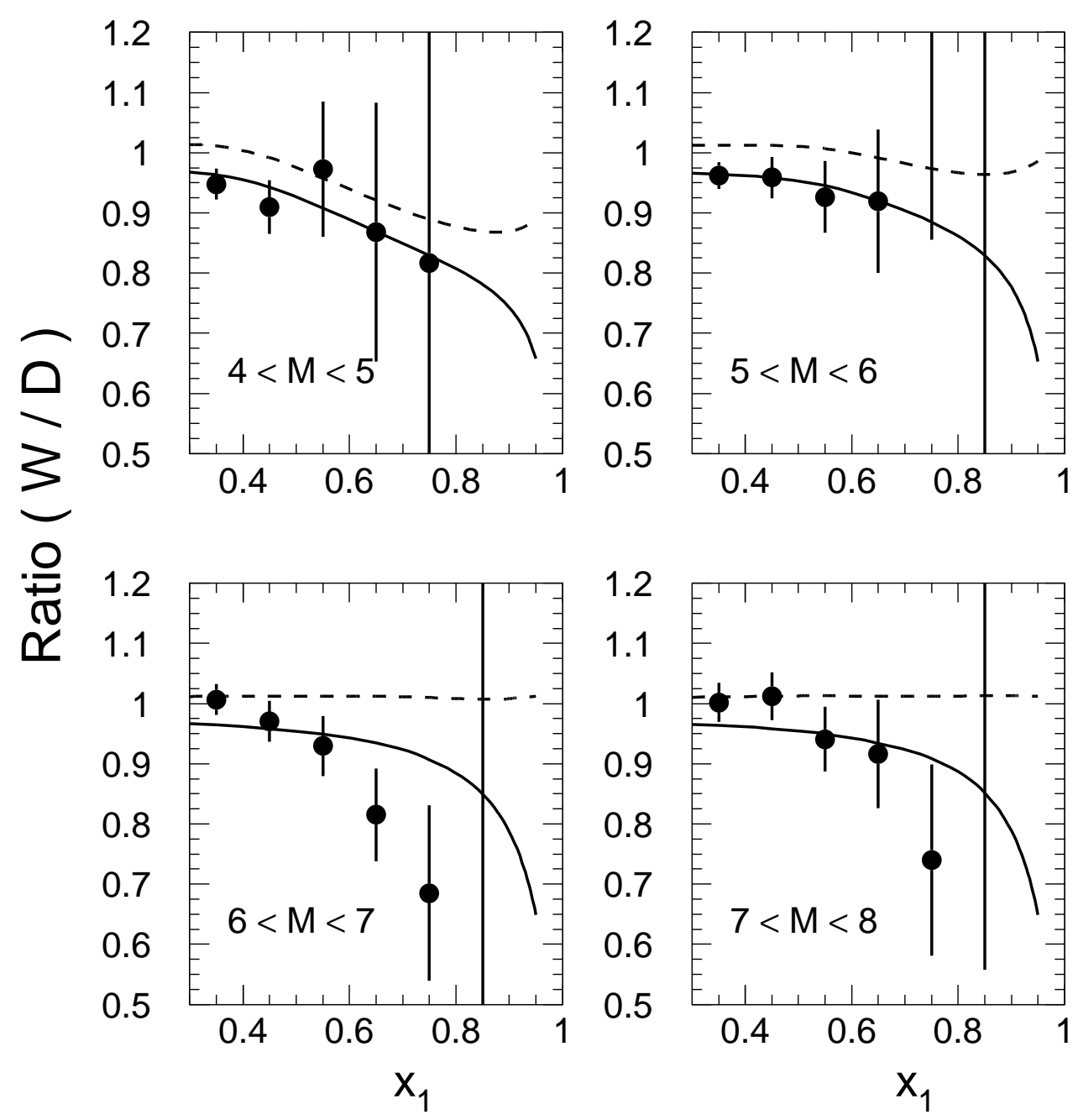

Figure 12: The same as Fig. 11, except that the ratio of tungsten to deuterium is shown.

by the string model. However the string tension, $\kappa_{s}=2 \pi \alpha_{R}^{\prime} \approx 1 \mathrm{GeV} / \mathrm{fm}$ is a static quantity, related to the slope parameter $\alpha_{R}^{\prime}$ describing the orbital excitation spectrum of hadrons. Evidently the energy loss by a quark includes an additional piece, such as the dynamical contribution arising from gluon radiation [9, 26]. The magnitude of the latter was determined in section 3.1 .2 to be similar in size to the energy loss arising from the formation of strings. Adding this contribution, the result (65) agrees with the expectation Eq. (31).

The normalization factors found for the E772 and E866 data are well within the quoted systematic errors. These values of norms explain why the dashed curves representing net shadowing in Figs. 11, 12 do not match unity for short coherence length. At the same time the dashed curves slightly rise above 1 at large $x_{1}$ and $M$, demonstrating antishadowing. This is a result of the delayed onset of shadowing toward small $x_{2}$ for heavy nuclei $(W)$ 

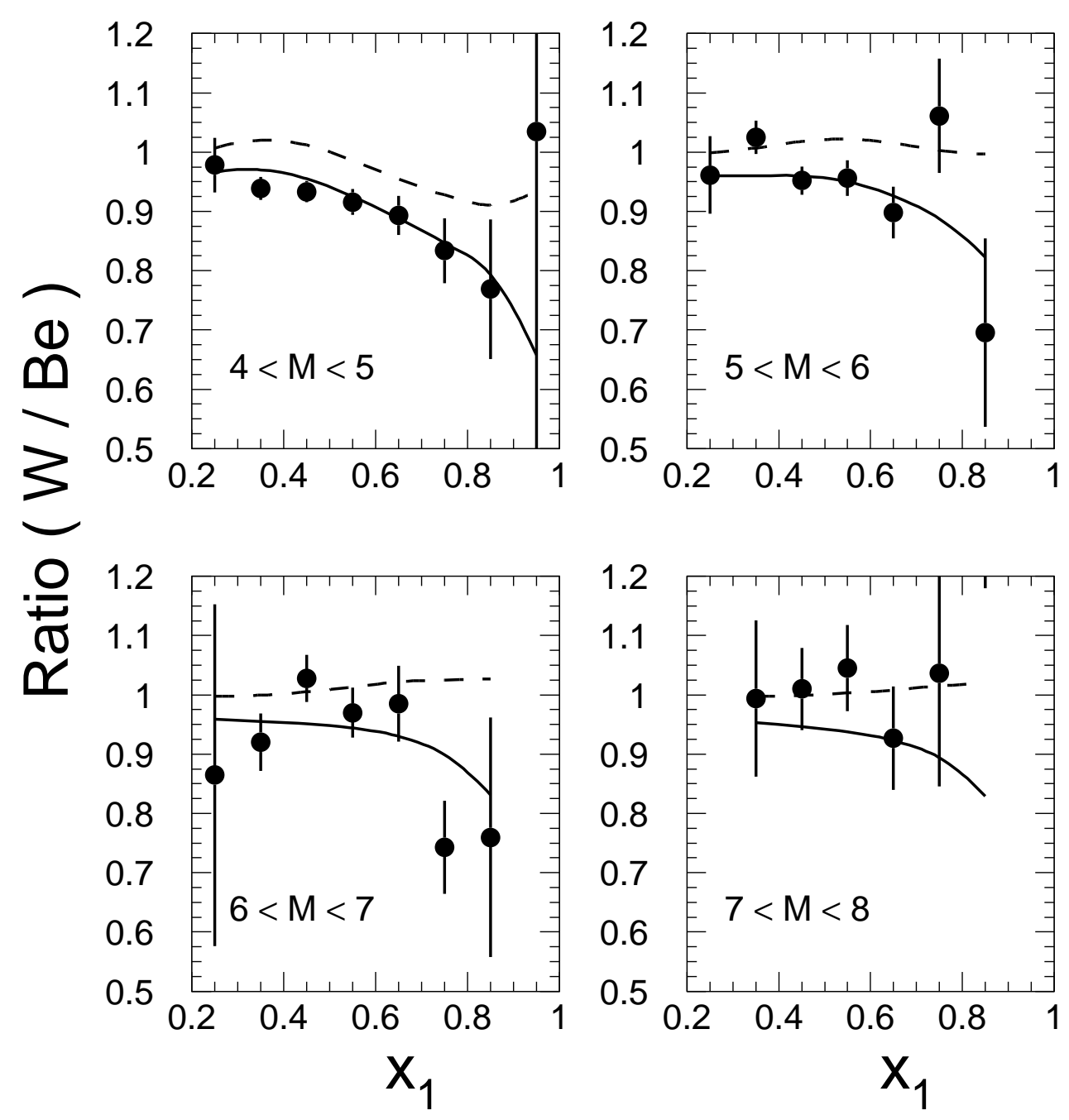

Figure 13: The same as Fig. 11, except that the ratio of tungsten to beryllium is shown, with data from Refs. [13], 477.

compared to light ones $(D)$ as dictated by their formfactors.

One can see from Figs. 11 - 13 that the effects of energy loss and shadowing display quite different behaviors as a function of $A, x_{1}$, and $M$. For carbon nearly all nuclear suppression, to the extent that it exists, comes from shadowing. This is because the mean path length available for energy loss in nuclear matter vanishes for nuclei as light as carbon, as one can see from Fig. 6. At the same time, for tungsten, energy loss makes a substantial contribution to nuclear suppression, while shadowing vanishes for large masses because the nuclear formfactor falls steeply at large $x_{2}$. This difference in the $A$ and $M$ dependence helps to disentangle the two effects rather effectively.

Another specific signature of energy loss is a suppression which does not vanish even at small $x_{1}$. Indeed, this effect of the order of $4 \%$ seems to be supported by data for tungsten in Figs. 12, 13, while data for carbon, Fig. 11 show no deviation from 1. This feature also 
helps to single out the effect of energy loss since the data have especially high accuracy in this region of small $x_{1}$.

Notice that the ratios of tungsten to beryllium shown in Fig. 13 reveal an interesting feature; namely, at large dimuon masses this ratio rises with $x_{1}$ reaching values above one, reminiscent of antishadowing. Instead of this, one might have expected a stronger shadowing for a heavier nucleus. Of course Eq. (54) cannot lead to any antishadowing. However, the onset of shadowing with decreasing $x_{2}$ must be delayed for heavier nuclei whose formfactors decrease more steeply with $q_{c}$. We illustrate this feature in Figs. 14 and 15, corresponding to the dimuon masses $M=4.5$ and $7.5 \mathrm{GeV}$, respectively. We compare nuclear shadowing
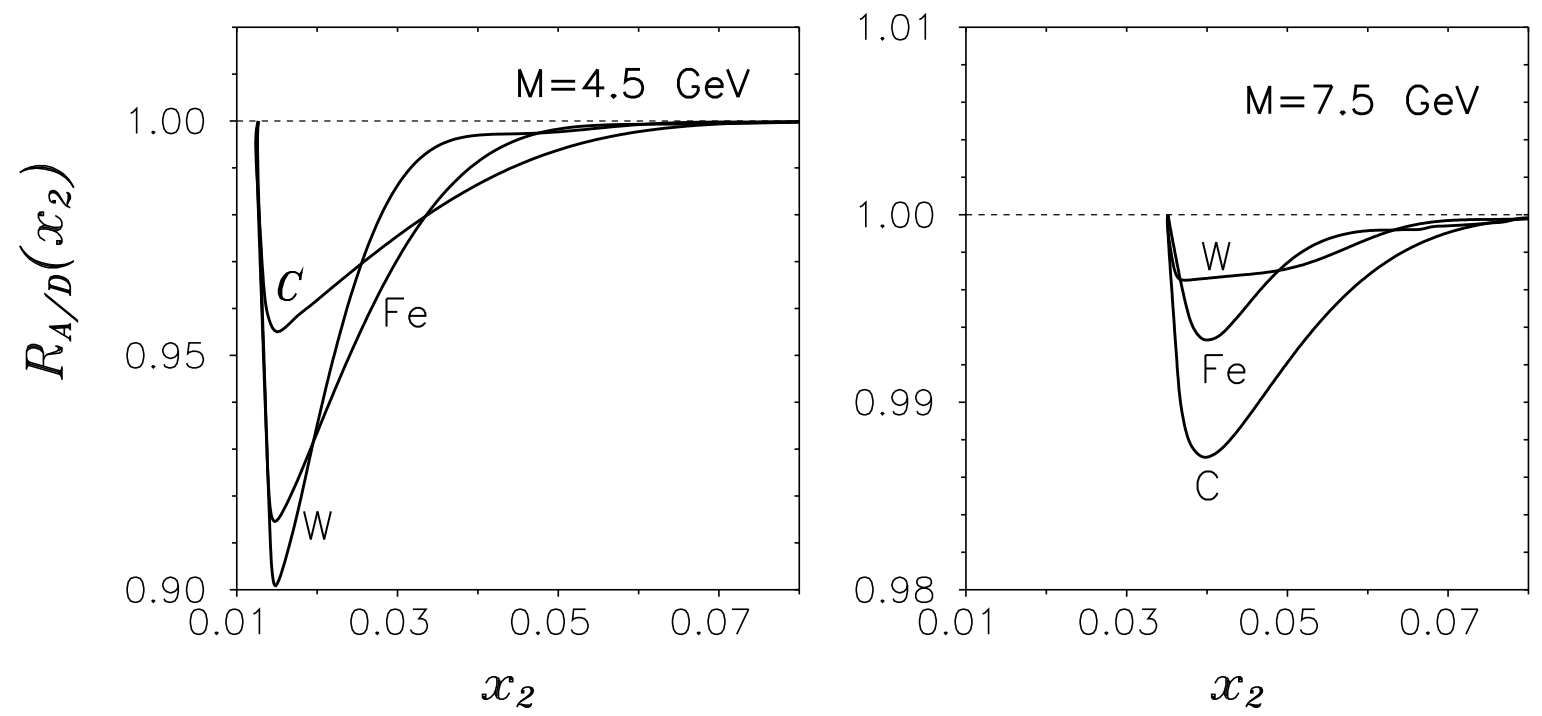

Figure 14: Shadowing in DY reaction on carbon, iron and tungsten as function of $x_{2}$ at $M=4.5 \mathrm{GeV}$. Nuclear shadowing disappears at large and small $x_{2}$ because the coherence length, Eqs. (7) and (8), vanishes in these limits.

Figure 15: The same as in Fig. 14, but at $M=7.5 \mathrm{GeV}$.

calculated with Eq. (54) for carbon, iron and tungsten as function of $x_{2}$. Indeed, one can see in Fig. 14 that the onset of shadowing for heavier nuclei occurs at smaller $x_{2}$ (compare with Figs. 5 and 6 in 25]). At the larger dimuon mass of $M=7.5 \mathrm{GeV}$ in Fig. 15, the behavior is seen to be even more complicated. In this case $l_{c}$ is so short that it suppresses the formfactor of tungsten at all $x_{2}$. In any case, these tiny variations of shadowing have no influence on our final results for energy loss.

Note that shadowing vanishes towards the kinematical limit $x_{1}=1$, i.e. minimal values of $x_{2}$. This is the result of the coherence length vanishing in this limit (see Fig. 3). This property is irrelevant for our analysis since there is no data in this region. 


\subsection{Stability of the solution}

We have performed several tests of the stability of our results given in (65) - (67). First of all, as already mentioned, the analysis could be affected by any overlooked physical effects that are related to nuclear structure and that exist at large $x_{2}$ where no shadowing is expected. To test this, we varied the relative number of shadowed and unshadowed events by imposing an upper cut-off $x_{2}^{\max }$ on the values of $x_{2}$ allowed in the data set. As this cut-off is lowered, more points are affected by shadowing. On the other hand, decreasing the cut-off $x_{2}^{\max }$ diminishes the influence of any missed physical effects, as mentioned above. We have plotted the values of the energy-loss rate $\kappa$ resulting from separate fits to the E772 and E866 data as a function of $x_{2}^{\max }$ in Fig. 16. The results of the fits to both sets of data appear

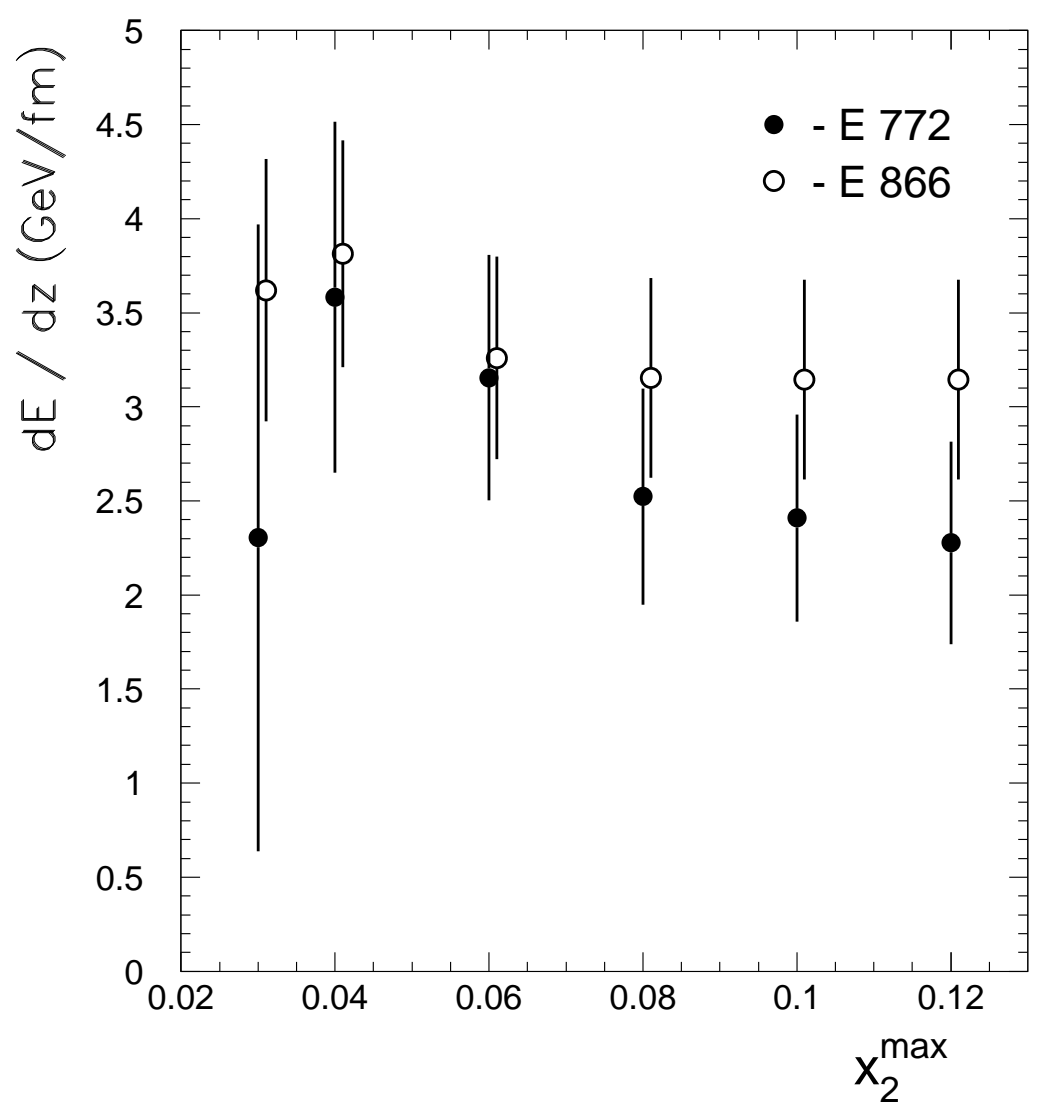

Figure 16: The rate of energy loss $-d E / d z=\kappa$ as a function of the upper cut-off $x_{2}<x_{2}^{\max }$. Closed and open circles correspond to fits to the E772 and E866 data respectively.

reasonably stable, confirming the correctness of our calculations for nuclear shadowing of the DY cross section.

Secondly, we tested the sensitivity of our results to the magnitude of the shadowing in the analysis. We replaced $1-R_{A / D}^{\text {shad }} \Rightarrow C_{\text {shad }}\left(1-R_{A / D}^{\text {shad }}\right)$, where the factor $C_{\text {shad }}$ was varied. 
Eliminating shadowing entirely by fixing $C_{\text {shad }}=0$, we found $-d E / d z=2.34 \pm 0.37 \mathrm{GeV} / \mathrm{fm}$ with $\chi^{2}=240$ from the common fit of the E772 and E866 data. Next, making another extreme assumption, we doubled the amount of shadowing by fixing $C_{\text {shad }}=2$ and found $-d E / d z=3.00 \pm 0.37 \mathrm{GeV} / \mathrm{fm}$ with $\chi^{2}=259$. Whereas one might be tempted to conclude from this exercise that the treatment of shadowing is unimportant the extraction of energy loss, one clearly sees from from Fig. 16 that this is not true: the energy-loss value determined by the data acquires important contributions from the shadowing region. We also checked the optimal value of $C_{\text {shad }}$ by treating it as a free parameter and found $C_{\text {shad }}=0.88 \pm 0.18$ with $\chi^{2}=218$, which confirms correctness of our calculations for shadowing. Thus, we conclude that by varying the amount of shadowing over a wide range, the rate of energy loss remains unchanged within error bars, while $\chi^{2}$ becomes substantially worse.

Next, we examined the accuracy of the double-scattering approximation in Eq. (54). We had assumed that if the shadowing effect is weak, we could neglect the triple and higher order rescattering terms. The exact calculation of these corrections in [23, 19] is too cumbersome to be used in the fitting procedure. Instead, one can include the multiple interaction corrections in the same way as in Ref. [44], i.e. assuming that rescatterings happen with the same cross section $\sigma_{\text {eff }}$ that governs the shadowing correction in (54). Then, the formfactor Eq. (55) should be modified as,

$$
\tilde{F}_{A}^{2}\left(q_{c}\right)=\frac{2}{\langle T\rangle} \int d^{2} b \int_{-\infty}^{\infty} d z_{2} \rho_{A}\left(b, z_{2}\right) \int_{-\infty}^{z_{2}} d z_{1} \rho_{A}\left(b, z_{1}\right) \exp \left[i q_{c}\left(z_{2}-z_{1}\right)-\frac{\sigma_{e f f}}{2} \int_{z_{1}}^{z_{2}} d z \rho_{A}(b, z)\right] .
$$

We repeated the fit replacing $F_{A}^{2}\left(q_{c}\right) \Rightarrow \tilde{F}_{A}^{2}\left(q_{c}\right)$ in Eqs. (54) and (64) and found no visible deviation from the result in Eq. (65).

It was also of interest to know whether our results would change if, instead of using Eq. (64) to combine the effects of shadowing and energy loss, we make the maximal-strength mixture of these two effects as assumed in [13]; namely, $R_{A / N}=R_{A / N}^{\text {Eloss }} R_{A / N}^{\text {shad }}$. Fitting all data when we do this, we find nearly the same value of the energy loss rate that we previously obtained, $-d E / d z=2.18 \pm 0.31 \mathrm{GeV} / \mathrm{fm}$.

One can also extract from the results of these modifications a scale for the model dependence of the systematics in the rate of energy loss. We evaluate such a systematic error at about $\pm 0.5 \mathrm{GeV} / \mathrm{fm}$. Note that although all these variations of the fitting procedure do not affect the results very much, they do substantially increase the value of $\chi^{2}$ compared to the fit done in Sect. 5.1.

The pleasing stability of the results confirms the conclusion of Sect. 5.1 that there should be no confusion between the effects of shadowing and energy loss (provided that the data are sufficiently exact and binned both in $x_{1}$ and $M$ ) since these two effects behave very differently as functions of $A, x_{1}$ and $M$.

Finally, we would like to call attention to the fact that the data we are analyzing for $\kappa$ cover the kinematical region of the target $x$ extending all the way out to large values of $x_{2}$. This is a concern, since for $x_{2}>0.1$ there exist EMC and antishadowing effects in nuclear quark distribution function that we have not yet accounted for in the theory. We suppress the contribution of large $x_{2}$ selecting data with $x_{1}>0.3$. Besides, we argue next that these 
effects make a negligible contribution to the data we are analyzing.

To show this, we first remark that the EMC/antishadowing effects appear in the quark distribution function of the nuclear target, corresponding to antiquarks in the proton projectile. We expect that the EMC/antishadowing corrections, if appreciable, can affect the analysis only at small $x_{1}$, since antiquarks in the beam contribute only for small $x_{1}$. These corrections enter the theory as expressed in Eq. (64) in both $R_{A / N}^{\text {shad }}\left(x_{1}, M^{2}\right)$ (Eq. (54)) and $R_{A / N}^{\text {Eloss }}\left(x_{1}\right)$ (Eq. (41)).

Consider first the shadowing contributions. Shadowing is appreciable only for very small $x_{2}<0.03$. Taking $s=1600 \mathrm{GeV}^{2}$, these small values of $x_{2}$ correspond to values of $x_{1}=$ $M^{2}\left(s x_{2}\right)^{-1}>0.3$ at the smallest values of $M$ in our data set. For $x_{1}>0.3$, the antiquark distribution function is quite small, and in any case $\sigma_{\text {eff }}$, Eq. (60), is not very sensitive to how we average. So, we can neglect the antiquarks in the shadowing region.

Consider next the energy-loss contribution. For E772, all points in the data set correspond to $x_{1}>0.3$. This means that the data points are uncontaminated by EMC and antishadowing effects (i.e., $x_{2}<0.1$ ) for values of $M<6.9 \mathrm{GeV}$. However, for larger values of $M$ in E772, and for all values of $M$ in E866, some region of $x_{1}$ will be affected. We have made quantitative estimates of the size of this contamination by comparing the quark and antiquark contributions to the integral in Eq. (41). We evaluated the antiquark contribution using the same the cross section $d \sigma_{D Y}^{q N}\left(x_{1}^{q}\right) / d x_{1}^{q}$ that we found for an incident quark, Eq. (45), and using the simple expression 48

$$
\bar{q}\left(x_{1}\right)=\frac{0.7(1-x)^{8}}{4 x}
$$

for the atiquark distribution. For $A=184$ and $\kappa=2.28 \mathrm{GeV} / \mathrm{fm}$, we find that the antiquark contribution is only $0.3 \%$ of the quark contribution at $x_{1}=0.1$. Additionally, the relative size drops rapidly for larger values of $x_{1}$, allowing us to conclude that the EMC/antishadowing effects make a tiny contribution to the DY cross section throughout the kinematical region we are considering and justifying our neglect of these effects in the data analysis.

\subsection{Why a previous analysis [13] found no energy loss}

One may wonder why the rate of energy loss $-d E / d z$ deduced from the E866 data in a previous analysis [13 is so much smaller $(-d E / d z<0.44 \mathrm{GeV} / \mathrm{fm})$ than the value we found fitting the same data. In this section we address this question. I A value smaller than the string tension $\sim 1 \mathrm{GeV} / \mathrm{fm}$ would be at the same time both surprising and a serious problem for the theory.

\footnotetext{
${ }^{2}$ We compare only with the second of three scenarios for energy loss considered in [13]. The first one (energy loss is proportional to the initial energy) violates the Landau-Pomeranchuk principle (see discussion in [12]), while the third version (induced energy loss) corresponds to energy loss induced by multiple interactions of a quark propagating through infinite nuclear matter [36]. However the dominant contribution comes from vacuum energy loss (see Sect. 3.1) which has a constant and energy independent rate and is properly treated with the second version considered in [13.
} 
One obvious reason for a difference is the effect discussed in Sect. 3.2 and Fig. 6, namely the observation that when the multiple scattering of the incident proton is properly considered, the mean path of a quark available for vacuum energy loss in nuclear matter is more than twice as short as it would be if such scattering were neglected. Taking this effect alone, we estimate that it would increase the $1 \sigma$ upper limit for the rate of energy loss found in Ref. [13] to $-d E / d z<1 \mathrm{GeV} / \mathrm{fm}$.

However, there are deeper reasons for the differences between the results of our analysis and that in Ref. [13], which treats the DY process as $q \bar{q}$ annihilation as in the leading-order parton model. These reasons are related to the fact that the parton model interpretation of the space-time development of the interaction is not Lorentz invariant and depends on the reference frame. As mentioned already in Sect. 2.1, the lack of Lorentz invariance arises because objects such as partons are quantum fluctuations rather than classical particles. Because of their quantum nature, the partons live and die differently in the different frames. Thus, one must avoid trying to look at the same parton in different reference frames, even though $x_{1}$ is invariant under relative Lorentz boosts. For example, if the DY process is treated in the lab frame, then the fraction of light-cone momentum carried by the valence quark, $x_{q}$, is larger than $x_{1}$ [see Eq. (9)]. However, in the dilepton rest frame $x_{q}=x_{1}$; this is no surprise since they are not the same parton!

\subsubsection{Advantages of Target Rest Frame Formulation}

From the above discussion, it is clear that a consistent approach to the calculation of energy loss would, in principle, have to treat all effects in the same frame. In the case of the standard parton model approach [1], this would be the rest frame of the dilepton. In this frame, the moving nucleus is subject to a contraction by the Lorentz $\gamma$-factor, $R_{A}^{*}=R_{A} / \gamma$, where

$$
\gamma=\frac{M}{2 x_{2} m_{N}} .
$$

The energy loss $\Delta E^{*}$ in this reference frame is diminished by the same factor compared to the one in the nuclear rest frame, $\Delta E^{*}=\Delta E / \gamma$. Indeed, the vacuum energy loss is medium independent and proportional to $L^{*}=L / \gamma$. The induced energy loss is proportional to the Lorentz boosted nuclear density $\rho_{A}^{*}=\gamma \rho_{A}$, but also to $\left(L^{*}\right)^{2}=L^{2} / \gamma^{2}$. At the same time, the cloud of antiquarks in the target nucleon spreads in longitudinal direction over distance $\sim 2 / M$. Thus at small $x_{2}$,

$$
x_{2} \ll \frac{1}{m_{N} R_{A}},
$$

(this is exactly the condition for long coherence length, i.e. for shadowing) the size of the antiquark cloud substantially exceeds the nuclear radius.

Unfortunately, in the rest frame of the dilepton the position of $\bar{q} q$ annihilation cannot be localized to better than $\sim 1 / M$, while the nucleus is squeezed by the Lorentz transformation to a pancake shape of a much smaller thickness $\sim x_{2} R_{A} m_{N} / M$. The annihilation process clearly lacks sufficient resolution to probe such small distances. Additionally, one cannot say whether it is the quark or the antiquark that propagates through the nucleus prior to 
annihilation, a distinction that obviously must be made even before considering the issue of energy loss.

For these reasons, it is quite tempting to switch reference frames (which, however, we have argued is invalid, unless it is done properly) and view the reaction from the rest frame of the nucleus. Just such a switch occurred in Refs. [11, 13]. For example, in Ref. [13] the shift $\Delta x_{1}$ caused by energy loss was first fit to the E866 data in the rest frame of the dilepton. The authors then switched to the rest frame of the nucleus, writing their Eq. (2) in this frame and assuming, in accordance with [33, 12], that $\kappa_{2}$ is a constant independent of $x_{1}, x_{2}$ and $s$. They find the rate of energy loss to be $\Delta E=\Delta x_{1} E /\left(3 R_{A} / 4\right)$, where $E=800 \mathrm{GeV}$ is the beam energy.

One might try to avoid these inconsistencies by treating the DY reaction as $q \bar{q}$ annihilation in the rest frame of the nucleus, imagining the projectile parton traveling through nuclear matter and suddenly annihilating with a sea antiquark in one of the bound nucleons. This is, however, problematic for several reasons. First of all, the sea antiquarks available for such annihilation are defined only in the infinite momentum frame (this is why Weitzäcker-Williams photons do not exist in the rest frame of the electron-they are its static field). Secondly, annihilation of a high-energy massless quark with an antiquark at rest to a dilepton of mass $M$ violates energy-momentum conservation. To fix this problem, one may introduce next-to-leading order corrections, i.e. gluon radiation. As some of these gluons are radiated in advance of the dilepton and some later, one cannot treat the DY reaction as instantaneous but rather must consider its space-time development. Unavoidably, one arrives at the picture employed in present paper, where the DY pair is treated on the same footing as the gluons, i.e. as bremsstrahlung.

One is thus led in a natural way to the target rest frame formulation, which clearly identifies the incident quark as the one that propagates through the nucleus and loses energy prior to the radiation of the $\bar{l} l$ pair. The relative contribution of shadowing and this energy loss is governed by the coherence length appearing as an argument of the longitudinal form factor in Eq. (64). Specifying this dependence is essential for the determination of the correct value of the rate of energy loss from the data. This is because many of the E866 data are located at small $x_{2}$, where the coherence length is relatively long (Fig.(3)), implying that shadowing dominates over energy loss in all but the heaviest nuclei. Ignoring this dependence on $x_{2}$ would thus tend to overemphasize shadowing and lead to a diminished rate of energy loss, just as in Ref. 13.

\subsubsection{Comparison of Results for Shadowing}

Still a substantial fraction of the E866 data is located at rather large $x_{2}$ where no shadowing is expected. For these events, another difference exists between Ref. [13] and our results. This can be traced to the fact that the shadowing correction in 113], which is taken from the phenomenological analysis by Eskola, et al. [14, is quite different from ours in this region. We compare our shadowing calculated for tungsten at $M=4.5$ depicted in Fig. 17 by the dashed curve, with the one from Ref. [14] (solid thin curve) that was used in Ref. [13] to correct the E866 data. One can see quite clearly the difference between these two prescriptions. 


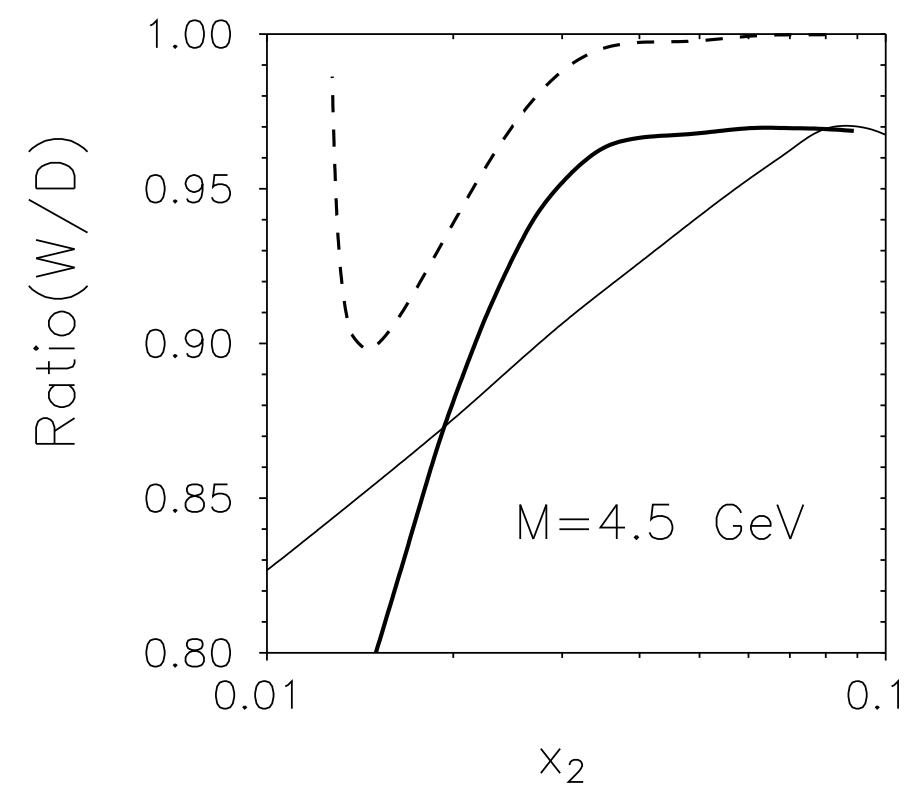

Figure 17: Comparison of nuclear shadowing (dashed curve) and combined effect of shadowing and energy loss (thick solid curve) presented in our analysis, with "shadowing" for antiquarks found in [14] from the combined analysis of DIS and DY data (thin solid curve). All calculations are done for tungsten and $M=4.5 \mathrm{GeV}$. The behavior of the dashed curve at small $x_{2}$ is explained in the text.

The source of the difference at large $x_{2}$ arose, we believe, from a confusion between energy-loss effects and shadowing in the phenomenological extraction of the antiquark densities in Ref. [14]. Shadowing for antiquarks cannot be extracted in a model independent way solely from DIS data, which are blind to the sign of the electric charge. This is why the E772 data for the DY reaction were included in the fit of Ref. [14 together with a variety of DIS data. Assuming QCD factorization, the evolution equations were applied to the parton distributions in nuclei. As usual, one needs to know the input $x$-distributions at a medium high scale $Q_{0}^{2}$. The shapes of these distribution were guessed in Ref. [14], and then their magnitude was fit to data. No physical input beyond the QCD evolution was incorporated in the analysis. It is not a surprise that substantial "shadowing" effects were found for antiquarks even at large $x_{2}$ where no quantum interference is possible because of the shortness of the coherence length. It was assumed in Ref. [14] that the ratio of the antiquark densities is constant for $x>0.08$. The magnitude of this ratio was fit to the E772 data and found to be less than 1, as one can see from Fig. 17. We think that this is where the misinterpretation of the energy loss effects as shadowing occurred in Ref. [14]. Indeed, as we emphasized in Sect. 5.1 and one can see in Figs. 11 - 13, energy loss suppresses the DY nuclear cross section even at small $x_{1}$ (i.e. large $x_{2}$ ) where no shadowing is possible. 
The thick solid curve in Fig. 17 representing our result for the combined effect of energy loss and shadowing confirms this conjecture. Indeed, it is below the shadowing (dashed) curve at large $x_{2}$ exactly by the same amount as the "shadowing" curve from Ref. [14].

The influence of energy loss on the effective nuclear suppression of antiquarks found in 114 extends down to smaller $x_{2}$ (where most of the E866 data are concentrated), giving to the $x_{2}$ distribution quite a different shape compared to the shadowing we calculated. In particular, nuclear suppression is strongest at the kinematical limit of smallest $x_{2}$ in the E772/E866 experiments, while shadowing shown by dashed curve vanishes in this limit. We should again emphasize that shadowing disappearing at small and large $x_{2}$ is a model-independent expectation since it is based only on the uncertainty principle and the kinematics of the DY reaction.

Although the nuclear suppression from [13 depicted in Fig. 17 by the thin solid line exceeds the calculated shadowing effect shown by the dashed curve even at small $x_{2} \sim$ $0.015-0.02$ this is not the reason for our disagreement with Ref. [13]. Indeed, as is stated above, we have checked that even with shadowing twice as strong as the one shown by the dashed curve in Fig. 17 (i.e. stronger at small $x_{2}$ than shadowing from Ref. [14]), the resulting energy loss is essentially the same. We suppose that the more important effect is the different shape suggested in [14] for the $x_{2}$ dependence of the nuclear suppression, which does not vanish at large $x_{2}$.

In conclusion, we believe that incorrect physical input at small $x_{2}$ and the particular antiquark density at large $x_{2}$ employed in the analysis of Ref. [13] led to an incorrect result of vanishing energy loss. Although this result was confirmed with much worse confidence by the recent analysis [49] of data from the NA3 experiment, it comes as no surprise that they arrived at the same conclusions since their analysis was done essentially the same way as that in [13].

\section{Summary and Conclusions}

We have presented a new analysis of the nuclear dependence of the E772/E866 Drell-Yan lepton pair production data. This analysis makes use of a new formulation of DY in the rest frame of the target, according to which the lepton pair arises from the decay of a massive photon radiated by the incident quark in a bremsstrahlung process. We have been particularly interested in these data as a source of information on the rate of energy loss of a quark propagating through nuclear matter, encouraged by the observation that the rate of fall off of the DY cross section data with $x_{1}$ near the point $x_{1}=1$ is extremely rapid and therefore sensitive to the amount of energy lost by the quark prior to radiating the photon. Since shadowing has a similar effect to that of energy loss, namely suppression of the cross section at small $x_{2}$, it is important that we have a good theoretical understanding of shadowing and that we explicitly use this understanding to model the shadowing.

Identification of the dominant physical processes, as well as the determination of their relative contribution, is guided by the notion of coherence time, which can be interpreted as the lifetime of a virtual fluctuation of the incident quark into the massive photon (and final 
quark). In particular, for short coherence times, the massive photon is released immediately from the fluctuation as the incident quark scatters from a bound nucleon. These processes clearly depend on the amount of energy lost by the soft incident quark as it propagates from its point of origin in a soft inelastic collision of the incident proton with a bound nucleon to the point at which it undergoes bremsstrahlung, and these processes are also sensitive to the energy lost by this quark for reasons given above. Because the coherence time is short, the photon is radiated immediately, and the final quark has no time to multiply scatter from other nucleons before the radiation process has come to completion. For long coherence times, the dominant processes correspond to terms in which the massive photon is radiated well in advance of any nuclear collision, and shadowing becomes an issue as the final quark begins to scatter from other nucleons of the nucleus. We describe shadowing quantitatively and without free parameters in terms of a phenomenological color-dipole cross section (fit to HERA data for the proton structure function), the nuclear thickness function, and the longitudinal nuclear form factor. Our model is shown to reproduce shadowing where it has been observed, namely in DIS on nuclei taken by the NMC collaboration. The transition between the regime of long and short coherence time is governed by the longitudinal form factor, with the longitudinal momentum transfer bearing a simple inverse relationship to the coherence length.

Our theory, as described above, contains one unknown parameter, namely the rate of energy loss $\kappa$ of a quark propagating through nuclei. Using $\kappa$ as a free parameter, the theory was fit to the E772/E866 data for DY lepton pair production arising from bombardment of ${ }^{2} \mathrm{H}$, Be, $\mathrm{C}, \mathrm{Ca}, \mathrm{Fe}$, and $\mathrm{W}$ targets by $800 \mathrm{GeV} / \mathrm{c}$ protons. The data were binned both in $x_{1}$ and $M$, a very important issue for reliable discrimination between the effects of energy loss and shadowing. We found $\kappa=2.28 \pm 0.31 \mathrm{GeV} / \mathrm{fm}$, in close accord with our theoretical estimate of $2 \mathrm{GeV} / \mathrm{fm}$ determined by considering string dynamics and gluon radiation. Numerous tests confirming the stability of our numerical analysis were made. They also provided a scale for the theoretical systematic uncertainty in the rate of energy loss, which we estimated at about $\pm 0.5 \mathrm{GeV} / \mathrm{fm}$. Since the value we found for $\kappa$ is substantially larger than the value reported in previous work, we pointed out the important differences between the two analyses.

One should be cautious determining nuclear shadowing of sea quarks from DY data which may be substantially contaminated by energy loss. An essential demonstration our conclusions requires similar data for the DY reaction on nuclei at lower energies, where shadowing is of no importance but energy loss produces a stronger effect on the cross section. On the other hand, at much higher energies of RHIC and LHC one can completely disregard energy loss and test models for shadowing by direct comparison to data. We expect spectacular shadowing effects producing a strong suppression of the DY cross section for a wide range of $x_{1}$.

Acknowledgment: We are grateful to Jörg Hüfner, Alberto Polleri, Jörg Raufeisen, Alexander Tarasov and Urs Wiedemann for numerous inspiring and clarifying discussions, and to Don Geesaman for valuable comments. This work has been partially supported by a grant from the Gesellschaft für Schwerionenforschung Darmstadt (GSI), grant no. GSI-OR-SCH. 


\section{References}

[1] S.D. Drell and T.M. Yan, Phys. Rev. Lett. 25 (1970) 316.

[2] L.S. Osborne et al., Phys. Rev Lett. 40 (1978) 1624.

[3] The EM Collaboration, J. Ashman et al., Z. Phys. C52 (1991) 1.

[4] The E665 Collaboration, M.R. Adams et al., Phys. Rev. D50 (1994) 1836.

[5] The HERMES Collaboration, A. Airapetian et al., Hadron Formation in Deep-Inelastic Positron Scattering in a Nuclear Environment, hep-ex/0012049.

[6] B.Z. Kopeliovich, J. Nemchik, E. Predazzi, Hadronization in Nuclear Environment, in the Proceedings of the Workshop on Future Physics at HERA, DESY, 1996 (nuclth/9607036); hep-ph/9511214.

[7] D.M. Alde et al., Phys. Rev. Lett. 66 (1991) 133.

[8] B.Z. Kopeliovich, A.V. Tarasov, J. Hüfner, Coherence Phenomena in Charmonium Production off Nuclei at the Energies of RHIC and LHC, hep-ph/0104256.

[9] B.Z. Kopeliovich and F. Niedermayer, Nuclear screening in $J / \Psi$ and Drell-Yan pair production, JINR-E2-84-834, Dubna 1984, a scanned version in KEK library: http://www-lib.kek.jp/cgi-bin/img_index?8504113.

[10] D.M. Alde et al., Phys. Rev. Lett. 64 (1990) 2479.

[11] S. Gavin and J. Milana, Phys. Rev. Lett. 68 (1992) 1834.

[12] S.J. Brodsky and P. Hoyer, Phys. Lett. 298B (1993) 165.

[13] M. Vasiliev et al., Phys. Rev. Lett. 83 (1999) 2304.

[14] K. J. Eskola, V. J. Kolhinen, and P. V. Ruuskanen, Nucl. Phys. B535 (1998) 351; K. J. Eskola, V. J. Kolhinen, and C.A. Salgado, Eur. Phys. J. C9 (1999) 61.

[15] M.B. Johnson, et al., Phys. Rev. Lett. 86 (2001) 4483 (hep-ex/0010051).

[16] B.Z. Kopeliovich, Soft Component of Hard Reactions and Nuclear Shadowing (DIS, Drell-Yan reaction, heavy quark production), in proc. of the Workshop Hirschegg'95: Dynamical Properties of Hadrons in Nuclear Matter, Hirschegg, January 16-21,1995, ed. by H. Feldmeier and W. Nörenberg, Darmstadt, 1995, p. 102 ( hep-ph/9609385).

[17] S.J. Brodsky, A. Hebecker and E. Quack, Phys. Rev. D55 (1997) 2584.

[18] B.Z. Kopeliovich, A. Schäfer and A.V. Tarasov, Phys. Rev. C59 (1999) 1609 (extended version in hep-ph/9808378). 
[19] B.Z. Kopeliovich, J. Raufeisen and A.V. Tarasov, Phys. Lett. B503 (2001) 91.

[20] N.N. Nikolaev and B.G. Zakharov, Z. Phys. C49 (1991) 607.

[21] Al.B. Zamolodchikov, B.Z. Kopeliovich and L.I. Lapidus, Sov. Phys. JETP Lett. 33 (1981) 612.

[22] S.J. Brodsky, A.H. Mueller, P. Hoyer, and W-K. Tang, Nucl. Phys. B369 (1992), 519.

[23] B.Z. Kopeliovich, J. Raufeisen and A.V. Tarasov, Phys. Rev. C62 (2000) 035204.

[24] K. Golec-Biernat and M. Wüsthoff, Phys. Rev. D59 (1999) 014017; hep-ph/9903358.

[25] B.Z. Kopeliovich, A. Schäfer and A.V. Tarasov, Phys. Rev. D62 (2000) 054022.

[26] B.Z. Kopeliovich and F. Niedermayer, Sov. J. Nucl. Phys. 42(1985) 504.

[27] A. Casher, H. Neuberger and S. Nussinov, Phys. Rev. D20 (1979) 179.

[28] B.Z. Kopeliovich, Phys. Lett. 243 B (1990) 141.

[29] A. Capella et al., Phys. Rep. 236 (1994) 225.

[30] A.B. Kaidalov, JETP Lett. 32 (1980) 474; Sov. J. Nucl. Phys. 33 (1981) 733; Phys. Lett. 116B (1982) 459.

[31] A.V. Abramovsky, V.N. Gribov and O.V. Kancheli, Yad. Fiz. 18 (1973) 595.

[32] B.Z. Kopeliovich, I.K. Potashnikova, B. Povh and E. Predazzi, Phys. Rev. Lett. 85 (2000) 507; Phys. Rev. D63 (2001) 054001.

[33] F. Niedermayer, Phys. Rev. D34 (1986) 3494.

[34] M. Gyulassy and M. Plümer, Nucl. Phys. B346 (1989) 1.

[35] J.F. Gunion and G. Bertsch, Phys. Rev. D25 (1982) 746.

[36] R. Baier, Yu.L. Dokshitzer, A.H. Mueller, S. Peigne and D. Schiff, Nucl. Phys. B483 (1997) 291; B484 (1997) 265; R. Baier, D. Schiff and B.G. Zakharov, Ann. Rev. Nucl. Part. Sci. 50 (2000) 37; U.A. Wiedemann and M. Gyulassy, Nucl. Phys. B582 (2000) 409.

[37] J. C. Peng, P. L. McGaughey, and J. M. Moss, talk presented at the RIKEN-BNL Workshop on Hard Parton Physics in Nucleus-Nucleus Collisions, March 1999, hepph/9905447

[38] H. De Vries, C.W. De Jager and C. De Vries, Atomic Data and Nucl. Data Tables, 36 (1987) 469. 
[39] R.J. Glauber, Phys. Rev. 100 (1955) 242; V. Franco and R.J. Glauber, Phys. Rev. 142 (1966) 1195.

[40] V. Anisovich at al., Phys. Let. 42B (1972) 224.

[41] P. L. McGaughey, et al., Phys. Rev. D 50 (1994) 3038.

[42] B. Z. Kopeliovich and B. Povh, Phys. Lett. B367 (1996) 329; Z. Phys. A356 (1997) 467.

[43] B.Z. Kopeliovich, J. Raufeisen and A.V. Tarasov, Phys. Lett. B440 (1998) 151.

[44] V. Karmanov and L. Kondratyuk, JETP Lett., 18 (1973) 451.

[45] The NMC Collaboration, P. Amaudruz et al., Nucl. Phys. B441 (1995) 3.

[46] Unpublished E772 data.

[47] Private Communication, E866 Collaboration.

[48] C. G. Whol, et al., Rev. Mod. Phys. 56 (1984) S1.

[49] F. Arleo, P.-B. Gossiaux, T. Gousset and J. Aichelin, Energy loss and $x_{2}$ scaling breakdown in $J / \psi$ nuclear production, hep-ph/0005230. 